\title{
An experimental study of nominally symmetric and asymmetric structures isolated with the FPS
}

\author{
Juan C. de la Llera*, ${ }^{*}$ and José L. Almazán \\ Department of Structural Engineering, Pontificia Universidad Católica de Chile, Casilla 306, Correo 22, \\ Santiago, Chile
}

\begin{abstract}
SUMMARY
This experimental investigation deals with the earthquake behaviour of a nominally symmetric and a mass-asymmetric three-storey structural model isolated with the frictional pendulum system (FPS). Both accidental and natural torsion are evaluated in the structure by using recorded accelerations in all building floors and measured deformations at the isolation level. A 3D-shaking table was used to subject the model to five different ground motions, including impulsive as well as far-field subductionzone type earthquakes. Results show that the analytical predictions of the earthquake behaviour of the isolated structure, as obtained from a physical model of the FPS, are in close agreement with the true complex inelastic measured behaviour of the FPS. Besides, experimental results also validate previous observations about the importance of accounting for the variability of the normal loads in modelling the earthquake behaviour of FPS isolators. Measured torsional deformation amplifications at the base of the building vary, in the mean, from $2.5 \%$ to $6 \%$ for the symmetric and asymmetric structural configurations, respectively. In relation to the fixed base structure, the reduction factors for the base shear of the isolated structure are, in the mean, about 3.9 for both configurations. Finally, it is concluded that the FPS is capable of controlling the lateral-torsional motions of mass-asymmetric structures quite effectively by aligning the centre of mass of the superstructure with the centre of pendular and frictional resistance of the isolation system. Copyright (C) 2003 John Wiley \& Sons, Ltd.
\end{abstract}

\section{INTRODUCTION}

Structures isolated with the frictional pendulum system (FPS) may undergo coupled lateral and torsional seismic motions as a result of accidental as well as natural factors. Accidental torsion in these structures is due mainly to variability in the isolator restoring forces caused by uncertainties in the sliding frictional behaviour, unforeseen variability in the isolator geometry, stiffness and mass eccentricities in the superstructure, rotational input, and overturning of the

* Correspondence to: Juan C. de la Llera, Department of Structural Engineering, Pontificia Universidad Católica de Chile, Casilla 306, Correo 22, Santiago, Chile.

† E-mail: jcllera@ing.puc.cl

Contract/grant sponsor: Chilean National Research Fund for Science and Technology, Fondecyt; Contract/grant number: 1000514

Copyright (c) 2003 John Wiley \& Sons, Ltd.

Received 19 January 2002

Revised 15 May 2002

Accepted 10 June 2002 
superstructure. Although this latter effect can be analytically predicted, it will be classified as an accidental factor since conventional small deformation structural models do not usually account for it.

Accidental torsion due to overturning of the superstructure is a consequence of bidirectional input motions. Since overturning increases the normal forces of some isolators and decreases the forces in others, a variation is observed in both, the pendular and the frictional components of the isolator restoring forces. Thus, a stiffness and a strength asymmetry results in the structure, in the same direction of motion as the one causing the overturning. Therefore, these eccentricities lead to coupled lateral and torsional motions only if the structure moves simultaneously in the perpendicular direction. In real earthquake motions, overturning occurs along both principal directions of the structure and, hence, stiffness and strength eccentricities occur along both directions.

On the other hand, natural torsion is caused by known mass and stiffness asymmetries at the isolation level and superstructure. The first question that arises is related to the kinematic admissibility of the motion of the isolation diaphragm supported on several isolators. Since the sliding interface is not planar and the motion of each isolator is constrained by the spherical surface, as large deformations occur, the isolation diaphragm will not remain plane and some of the isolators may undergo uplift. Thus, it is necessary that an FPS model capable of accounting for uplift and impact be used in the study. Another question is how well can the FPS control lateral-torsional coupling of a structure with plan asymmetry; and which should be the isolation parameters in order to control the response of the superstructure. As with conventional structures, accidental and natural torsion in an asymmetric structure cannot be evaluated independently.

In the literature, several analytical and experimental investigations have demonstrated the efficacy of the FPS system in reducing the translational seismic response of a structure [1-4]. For instance, experimental results obtained earlier have shown that isolated structures with the FPS may undergo an earthquake 4 to 6 times larger than their fixed-base counterparts while keeping its response within the elastic range [4]. More recently, a physical model has been proposed to evaluate large deformation effects in the FPS potentially leading to problems such as uplift and impact of the structure $[5,6]$.

So far, however, only few analytical as well as experimental investigations have focused in lateral-torsional coupling of structures isolated with the FPS. An early research $[1,2]$ dealt with structural models-length scales $E_{\mathrm{L}}=1 / 4$ and $1 / 9$ - of a two-storey structure with 4 FPS isolators located on top of the first-storey columns. Results of this study showed that the FPS system was able to reduce torsional effects for buildings with mass and stiffness eccentricity. In spite of the promising behaviour of the FPS in that case, there are some aspects that still need to be considered further before extending these conclusions to other buildings. First, the isolation layout in plan used in that research led to an uncoupled torsional-to-lateral frequency ratio $\Omega_{b}$ close to $\sqrt{3}$, i.e. a torsionally stiff system and hence, larger torsional responses might occur in structures with smaller frequency ratio of the isolation system, $\Omega_{b}$. Second, only a single ground motion component was used in the experimental study, which implied to neglect the accidental torsion effects caused by the overturning of the superstructure. And third, these experimental results should be used to validate the proposed large deformation models of the FPS system.

Consequently, the objectives of this experimental research are to: (i) develop an experimental program to evaluate accidental and natural torsion effects in structures isolated with the 
FPS; (ii) validate different aspects of the analytical FPS models used to predict the response of these structures; and (iii) evaluate the efficacy of the FPS in reducing the torsional response of nominally symmetric and asymmetric structures. It is intended also that the experimental results obtained from this study may serve as a benchmark for later analytical studies of lateral torsional coupling of structures with the FPS. Because similar results have been obtained for the cases of stiffness and mass asymmetry [6], this research will just focus in the case of mass asymmetry in the superstructure.

\section{EXPERIMENTAL SETUP AND TESTING PROGRAM}

The structural model used in this study corresponds to a three-storey frame building with two identical bays in the $X$-direction and three identical bays in the $Y$-direction with span length of $400 \mathrm{~mm}$ (Figure 1). The model was built in duralumin in a geometric scale $E_{\mathrm{L}}=1 / 7$ and to preserve an acceleration scale $E_{\mathrm{A}}$ equal to $1\left(E_{\mathrm{A}}=E_{\mathrm{L}} / E_{\mathrm{T}}^{2}=1\right)$, time had to be scaled down in the model by a factor $\sqrt{7}\left(E_{\mathrm{T}}=\sqrt{E_{\mathrm{L}}}\right)$. Beams and columns are hollowed square box shapes of sides 30 and $40 \mathrm{~mm}$, respectively, and $1.5 \mathrm{~mm}$ thickness. To enforce rigidity in the beamcolumn connections, a perforated steel core was introduced inside the beams and columns and it was bolted to L-shaped stiffeners placed at the bottom and top faces of the beam. The floor diaphragm is formed by 6 identical square steel plates of side $350 \mathrm{~mm}$, thickness $25 \mathrm{~mm}$, and weight $240 \mathrm{~N}$. These plates were bolted in each corner of its corresponding quadrant to a horizontal flange solidary to the L-shaped stiffeners. The building has a steel base slab of plan dimensions $1000 \mathrm{~mm} \times 1400 \mathrm{~mm}$ and a thickness of $13 \mathrm{~mm}$ (Figure 1). The total weight of the structural model is $W_{\mathrm{T}}=7540 \mathrm{~N}$, approximately.

The isolation system is composed of 4 FPS isolators with identical radius of curvature $R_{0}=143 \mathrm{~mm}$, i.e. a radius of $1000 \mathrm{~mm}$ in the prototype, or a nominal isolation period for the model of $0.76 \mathrm{~s}$, i.e. $2 \mathrm{~s}$ for the prototype. The deformation capacity of the isolators in the model is $50 \mathrm{~mm}$ ( $350 \mathrm{~mm}$ in the prototype). Shown in Figure 2 is a section of the isolator used and its principal dimensions. Perhaps one interesting feature of the isolator is that the slider was entirely made out of Teflon, as opposed to the more usual case in which a steel slider is coated with a Teflon layer. The four isolators were placed in the structure in inverted position as shown by Figure 2.

The model was instrumented with 19 acceleration channels, 6 placed on the moving platform to measure the input motions and 13 in the superstructure to measure floor accelerations. Besides, 3 additional linear potentiometers were placed between the moving platform and the base of the building to measure the relative displacements between the platform (ground) and the building. The physical location of the sensors in the model is indicated in Figure 1; their layout enables us to compute all floor accelerations in the two principal directions of the structure and the rotational motion of each floor relative to a vertical axis. These accelerations will be later used to compute storey shears and torques as well as response amplifications due to lateral-torsional coupling.

The shaking-table used is a 6 degree-of-freedom flight simulator which was adapted in 1998 by this research group as a shaking table. The simulator was manufactured by MOOG and is based in the original idea proposed by Stewart [7]. The six electro-mechanic actuators each control a single degree of freedom, which by a nonlinear kinematic transformation enable to control the three translational coordinates of the platform and the three angles of rotation, 


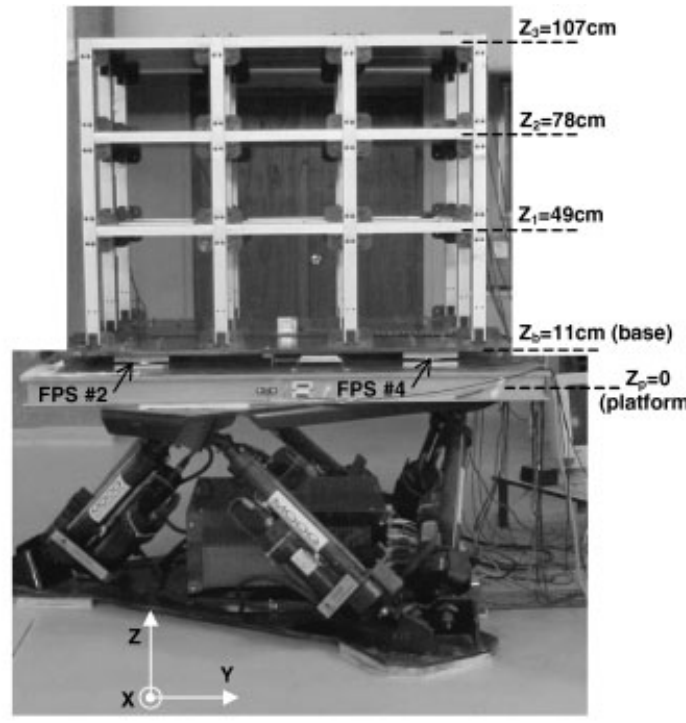

(a)

(c)

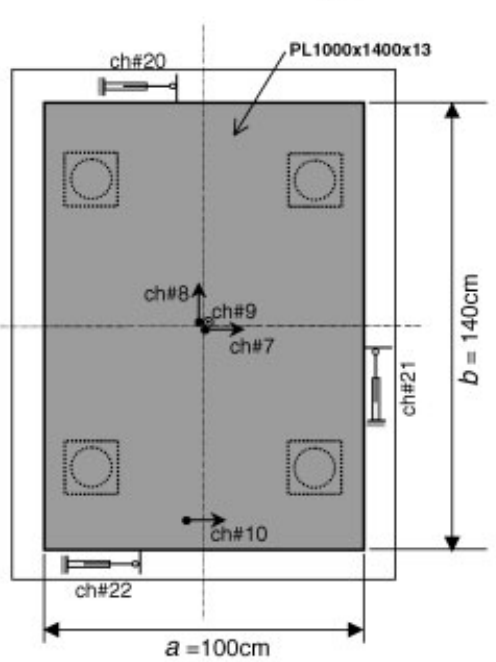

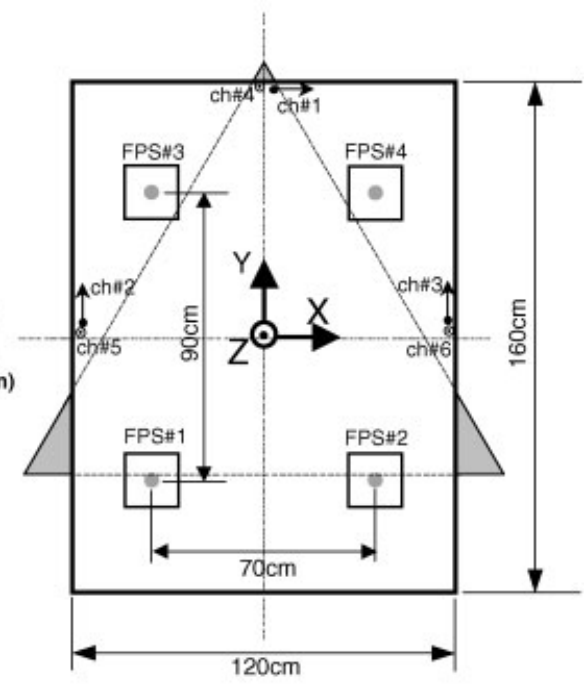

(b)

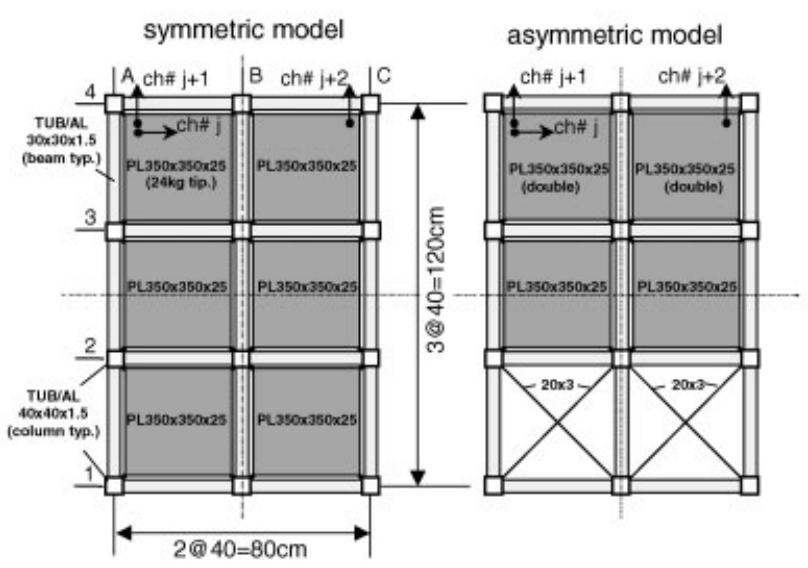

(d)

Figure 1. Experimental model considered: (a) front view; (b) planar view of the platform; (c) isolation base; and (d) symmetric and asymmetric model considered.

pitch, yaw, and roll. In this version, the platform and actuators are able to support a maximum load of $15 \mathrm{kN}$, approximately. Besides, the limit displacements, velocities, and acceleration presented in Figure 3.

The moving platform is identified in this figure by the vertices $A_{1}, B_{1}$, and $C_{1}$, into which the actuators $\mathrm{A}$ to $\mathrm{F}$ meet in pairs. Each actuator is connected to the base of the simulator by 

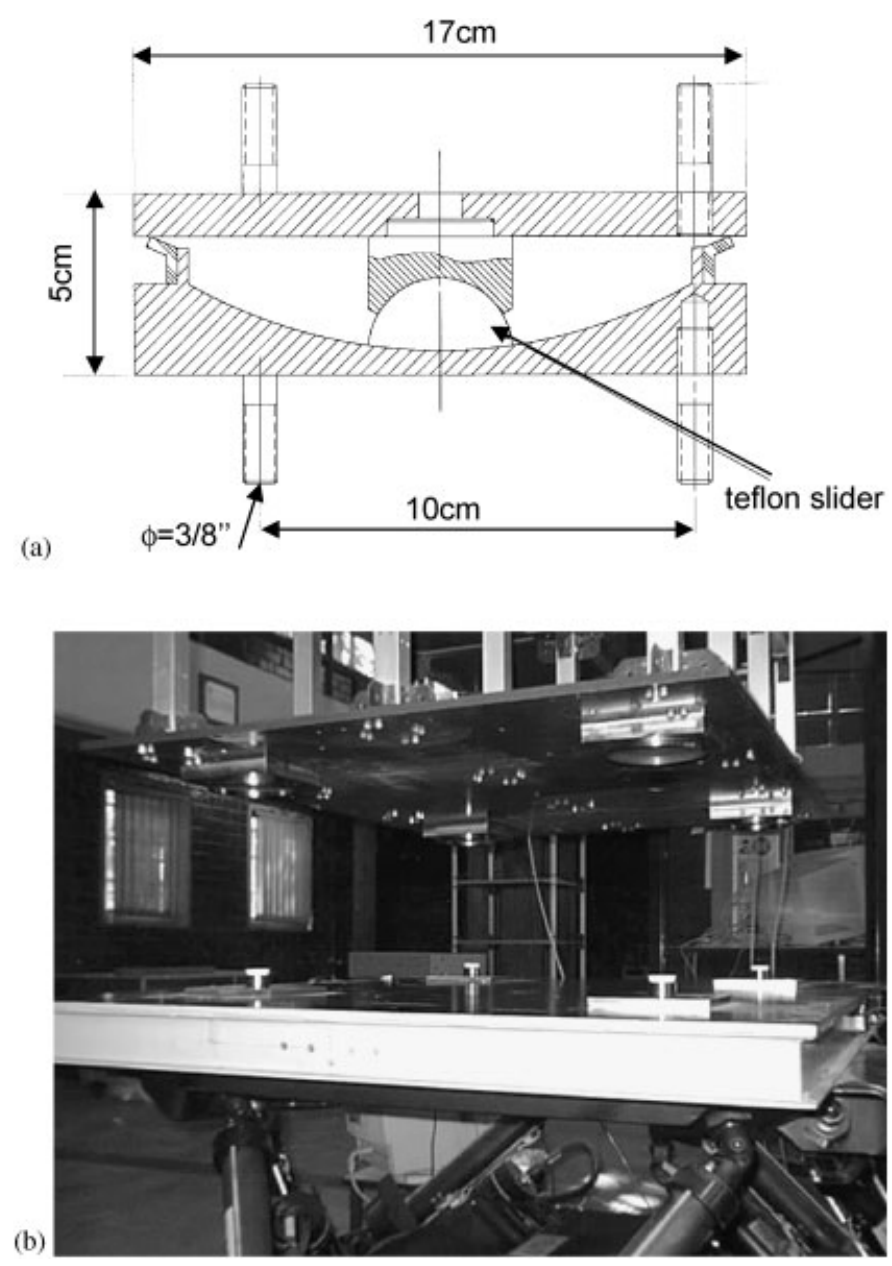

Figure 2. Isolation system: (a) FPS isolator dimensions; $y$ (b) isolators mounted in the structure and platform in inverted position.

spherical joints, describing in plan the triangular geometry described by vertices $A_{2}, B_{2}$, and $C_{2}$. Both triangles, which are equilateral, are rotated 60 degrees from each other and have three vertical planes of symmetry (Figure 3). A conventional PID-control is used for each actuator, which length must be transferred from an external computer in 20-byte frames to the computer in the platform in a RS-485 protocol at a sampling rate of $60 \mathrm{~Hz}$, i.e. a sampling time $h=0.017 \mathrm{~s}$. Sequence control of the degrees of freedom of the platform according to an earthquake ground displacement was developed and programmed in the Labview [8] environment; in order to achieve real-time tests, data acquisition was performed in parallel by using the software MTRACE [9].

The experimental investigation was divided into three phases. The earthquake response of the isolated base was studied first by considering a symmetric and asymmetric mass 


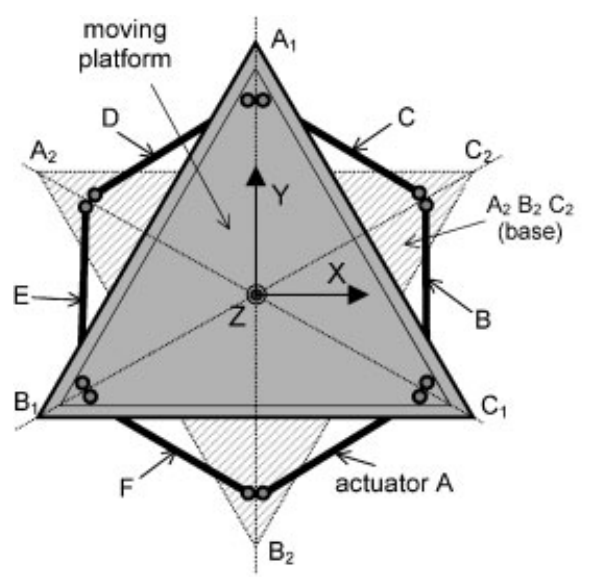

\begin{tabular}{|l|c|c|c|c|}
\hline \multirow{2}{*}{$\begin{array}{c}\text { Degree of } \\
\text { freedom }\end{array}$} & \multicolumn{2}{|c|}{ Displacement } & \multirow{2}{*}{ Velocity } & \multirow{2}{*}{ Accel. } \\
\cline { 2 - 3 } & Single & Comb. & & \\
\hline Lateral (X) & $\pm 23 \mathrm{~cm}$ & $\pm 28 \mathrm{~cm}$ & $\pm 50 \mathrm{~cm} / \mathrm{s}$ & $\pm 0.6 \mathrm{G}$ \\
\hline Frontal (Y) & $\pm 22 \mathrm{~cm}$ & $\pm 30 \mathrm{~cm}$ & $\pm 50 \mathrm{~cm} / \mathrm{s}$ & $\pm 0.6 \mathrm{G}$ \\
\hline Vertical (Z) & $\pm 18 \mathrm{~cm}$ & $\pm 18 \mathrm{~cm}$ & $\pm 30 \mathrm{~cm} / \mathrm{s}$ & $\pm 0.5 \mathrm{G}$ \\
\hline X-Rotation & $\pm 18^{\circ}$ & $\pm 24^{\circ}$ & $\pm 30^{\circ} / \mathrm{s}$ & $\pm 500 \% \mathrm{~s}^{2}$ \\
\hline Y-Rotation & $\pm 18^{\circ}$ & $\pm 22^{\circ}$ & $\pm 30^{\circ} / \mathrm{s}$ & $\pm 500 \% / \mathrm{s}^{2}$ \\
\hline Z-Rotation & $\pm 21^{\circ}$ & $\pm 23^{\circ}$ & $\pm 40^{\circ} / \mathrm{s}$ & $\pm 400 \% / \mathrm{s}^{2}$ \\
\hline
\end{tabular}

Figure 3. Schematic plan-view of the simulator and physical limits of degrees of freedom.

configuration. At this phase, special emphasis was placed in the identification of the 'true' frictional properties and behaviour of the isolation system. Second, the earthquake behaviour of the isolated building with symmetric and asymmetric mass distributions in the superstructure was considered (part (d) of Figure 1). Please notice that in the asymmetric mass configuration the two plates located between axes 1 and 2 were translated to the span between axes 3 and 4. Furthermore, to maintain an in-plane rigid diaphragm, L-shaped bracings in each quadrant were placed as a diaphragm between axes 1 and 2 . The mass eccentricity resulting from this asymmetric configuration is $e \approx 0.14 b$. In this phase, experimental results were contrasted with theoretical estimations obtained from the physical model of the FPS recently proposed $[5,6]$. In the third phase, the efficiency of the FPS for reducing the increase in deformation due to accidental and natural factors of torsion was evaluated and compared with the corresponding amplifications for fixed-base structures.

The testing program considered five three-component earthquake records that covered from near-field ground motions up to far-field subduction type ground motions: Newhall (Northridge, 1994; PGA =0.59 g), JMA (Kobe, 1995; PGA =0.83 g), El Centro (Imperial Valley, 1940; $\mathrm{PGA}=0.31 \mathrm{~g})$ amplified by a factor of $2(2 \times$ El Centro $)$, Melipilla (Chile, 1985; PGA $=0.69 \mathrm{~g})$ amplified by a factor of $(2 \times$ Melipilla), and a synthetic ground motion (Synthetic \#1) compatible with the design spectrum for seismic zone III and soil profile II (stiff soil) proposed for the new Chilean building code of base isolated structures (PGA $=0.66 \mathrm{~g}$ ). Also, to improve the identification of the isolation and building properties, other sinusoidal and pseudo white-noise excitations were used.

\section{RESPONSES CONSIDERED}

Response values defined next are computed directly from recorded accelerations and displacements by using simple equilibrium and kinematic equations [6]. These responses are: (i) the lateral deformations between the $\mathrm{CM}$ of the base slab and the ground (platform), $q_{x}$ and $q_{y}$; (ii) the total restoring force of the isolation system, $\mathbf{Q}_{b}$; (iii) the deformations of the 
building edge relative to the ground due to plan rotation only, $\delta_{\theta}^{(0)}=q_{\theta} b / 2$, where $b$ represents the length of the base (Figure 1); (iv) the $X$-direction total deformations of the edges of the building relative to the ground, $\delta_{ \pm b / 2}^{(0)}=q_{x} \mp \delta_{\theta}^{(0)}$; and (v) the $j$ th storey shear in the $X$-direction, $V_{x}^{(j)}$, and torque $T^{(j)}$.

To quantify the torsional effects in the structure, two global parameters are defined. These are the 'type- $\Xi$ ' parameters, which represent a quotient between the peak response at the edges of the plan due to plan rotation only and translation measured at the CM. For instance, $\Xi^{(0)}$ is defined as the percentual ratio between the deformation at the edge of the building due to rotation only and the lateral deformations measured at the $\mathrm{CM}$, i.e.

$$
\Xi_{b / 2}^{(0)}=100 \times \frac{\max \left\|\delta_{\theta}^{(0)}\right\|}{\max \left\|q_{x}\right\|}
$$

The second class of parameters are named 'type- $\Gamma$ ' and represent a quotient between the total peak response at the edges of the building plan and the peak responses at the CM. For instance, $\Gamma_{ \pm b / 2}^{(0)}$ is computed as the difference between the maximum total base deformation at the edge of the building relative to the base deformation at the CM, i.e.

$$
\Gamma_{ \pm b / 2}^{(0)}=100 \times \frac{\max \left\|\delta_{ \pm b / 2}^{(0)}\right\|-\max \left\|q_{x}\right\|}{\max \left\|q_{x}\right\|}
$$

Furthermore, the maximum $\Gamma$-response at the base will be denoted as $\Gamma_{b / 2}^{(0)}$, i.e.

$$
\Gamma_{b / 2}^{(0)}=\max \left(\Gamma_{+b / 2}^{(0)}, \Gamma_{-b / 2}^{(0)}\right)
$$

Please note that according to their definition, the type- $\Xi$ parameters are always an upper bound of the type- $\Gamma$ parameters; the two coincide if and only if the peak translational and rotational responses occur simultaneously.

Finally, a comparison is presented between the torsional amplifications obtained for the structural model with fixed and isolated base. The objective of these comparisons is to evaluate the capacity of the FPS system to control the torsional response of the superstructure caused by accidental and natural factors. Although floor deformations are readily available by double integration of the recorded floor accelerations, it was decided to evaluate superstructure responses only in terms of floor accelerations, shears, and torques to avoid introducing approximations in the response ratios presented. Notice that storey shears and torques are computed accurately since the building masses are known. Therefore, the following storey shear and torque reduction factors were defined for the superstructure:

$$
\mathrm{SRF}^{(j)}=\frac{\left(V_{x}^{(j)}\right)_{0}^{\mathrm{FB}}}{\left(V_{x}^{(j)}\right)_{0}^{\mathrm{IB}}} \quad \text { and } \quad \operatorname{TRF}^{(j)}=\frac{\left(T^{(j)}\right)_{0}^{\mathrm{FB}}}{\left(T^{(j)}\right)_{0}^{\mathrm{IB}}} \quad j=1: 3
$$

where the superindexes FB and IB denote the fixed-base and base isolated cases, respectively, and the quantities $\left(V_{x}^{(j)}\right)_{0}$ and $\left(T^{(j)}\right)_{0}$ represent the peak storey shears and torque values for the $j$ th storey, respectively. 


\section{SEISMIC BEHAVIOUR OF THE ISOLATED BASE}

The first step in this experimental investigation was to study accidental torsion due to unforeseen variabilities of the geometry and properties of the isolation system. This was done by removing the building from the isolated base. Thus, all uncertainties coming from the superstructure, most notably, the accidental torsion caused by the overturning of the superstructure were eliminated. The most relevant sources of plan asymmetry attributed to the isolation interface are: (i) variability in the friction coefficient of the isolators, (ii) imperfections in the spherical sliding surface, (iii) rotational input of the building model due to small control errors in the shaking table, and (iv) small errors in vertical alignment between the centre of the sliders and sliding surfaces. Please note that for structures isolated with the FPS, the centre of mass (CM) and the centre of the pendular action (CS) of the isolation system coincide at all times; hence, mass and stiffness eccentricities of the isolation interface are not a source of accidental torsion. Although little is known about the importance of these sources of accidental torsion in the building response, experimental results demonstrate that the major source of uncertainty is the variability in the friction coefficient of isolators.

As it was explained above, the building model was dismounted from the isolated base and replaced by two additional weights of $480 \mathrm{~N}$ each (Figure 4 ). The weights were placed in symmetric and asymmetric plan configurations, the latter with a mass eccentricity $e_{y}^{(m)}=15.9 \mathrm{~cm}$ in the $Y$-direction, or $e_{y}^{(m)}=0.113 b$. The nominal translational periods in both directions are $T_{x}=T_{y}=0.756 \mathrm{~s}$, while the nominal ratios between the translational and rotational periods are $\Omega_{b}=1.15$ and $\Omega_{b}=1.09$ for the symmetric and asymmetric models, respectively. These ratios were computed as a quotient between the radius of gyration of the stiffness and mass with respect to the $\mathrm{CM}$.

Consider as an introductory result the $X$-direction response of the base subject to the $\mathrm{N}-\mathrm{S}$ component of the Newhall record shown in Figure 5. The plot on the left shows the
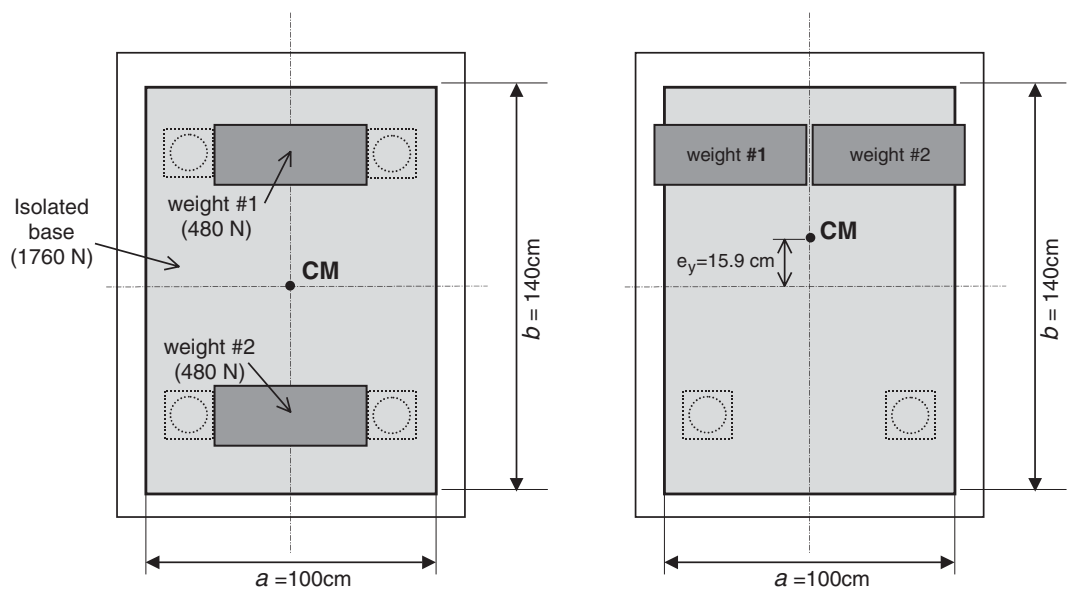

Figure 4. Symmetric and asymmetric experimental models considered for the base without the building. 

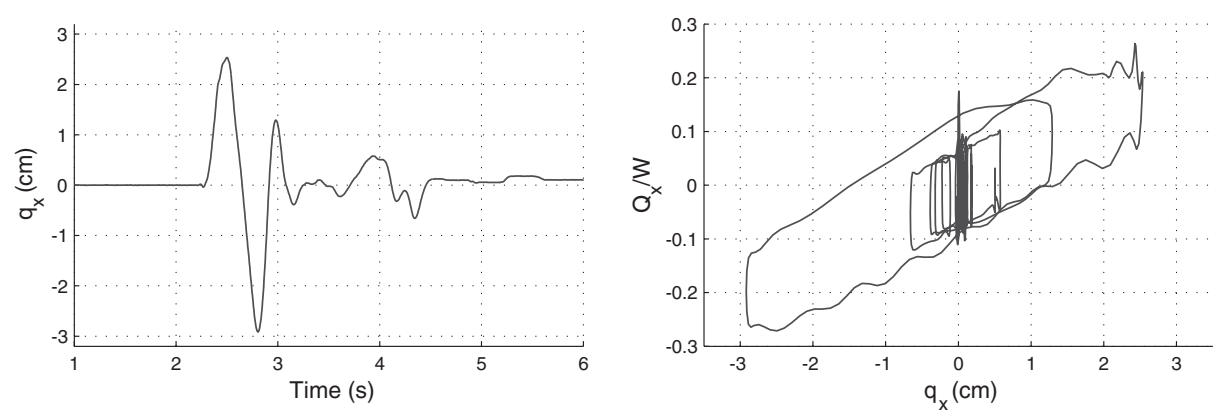

Figure 5. Earthquake response of the base with symmetric mass subject to the $\mathrm{N}-\mathrm{S}$ component of the Newhall record in the $X$-direction.

deformation of the base relative to the ground $q_{x}$, while the right plot shows the measured normalized force-deformation relationship of the isolation system, $Q_{x} / W$. Peak isolation deformations are about $3 \mathrm{~cm}$, i.e. $21 \mathrm{~cm}$ in the prototype, and occur for a single cycle during the strong initial part of the record. As a result of this motion the isolation system develops a maximum base shear of $26 \%$ of the weight of the structure. The shape of this experimental force-deformation loop validates the usual bilinear FPS model that combines the frictional and pendular actions. The loop shows also a significant sticking effect before the structure starts to slide and a clear dependency of the frictional force with velocity; more on the frictional behaviour will be presented next. Another interesting feature of this force-deformation cycle are the ripples observed in the loop due to the variation of the normal forces in the isolators.

A hybrid analytical-experimental methodology was established to estimate the frictional forces at the isolation interface from measured results. The idea is to subtract from the 'measured' total isolation restoring force $\mathbf{Q}_{b}=\mathbf{Q}_{b}^{(\mathrm{p})}+\mathbf{Q}_{b}^{(\mu)}$, an estimate of the pendular component of the restoring force $\mathbf{Q}_{b}^{(\mathrm{p})}$ given by $[6,10]$

$$
\mathbf{Q}_{b}^{(\mathrm{p})}=\mathbf{K}_{b} \mathbf{q}_{b}
$$

where $\mathbf{q}_{b}$ are the measured isolator deformations and $\mathbf{K}_{b}$ represents the stiffness matrix associated with the pendular action

$$
\mathbf{K}_{b}=\frac{\sum N_{k}}{R_{0}}\left[\begin{array}{ccc}
1 & 0 & -e_{\mathrm{p} y} \\
0 & 1 & e_{\mathrm{p} x} \\
-e_{\mathrm{p} y} & e_{\mathrm{p} x} & \rho_{\mathrm{p}}^{2}
\end{array}\right]
$$

and $\sum N_{k}$ is the sum of all normal forces in the isolators; $e_{\mathrm{p} x}=\sum N_{k} x_{k} / \sum N_{k}$ and $e_{\mathrm{p} y}=\sum N_{k} y_{k} /$ $\sum N_{k}$ are the stiffness eccentricities associated with the pendular action in the $X$ - and $Y$ directions, respectively; $\rho_{\mathrm{p}}^{2}=\sum N_{k}\left(x_{k}^{2}+y_{k}^{2}\right) / \sum N_{k}$ is the square of the radius of gyration of the pendular component; and $R_{0}$ is the radius of curvature of the FPS isolators. If the normal forces in the isolators are known, the pendular component of the restoring force would be determined accurately. For the sake of brevity, the equations used to produce a good analyticalexperimental estimate of the normal force are skipped in this presentation [6]. Once the frictional component of the restoring force $\mathbf{Q}_{b}^{(\mu)}$ is determined, the procedure developed will 

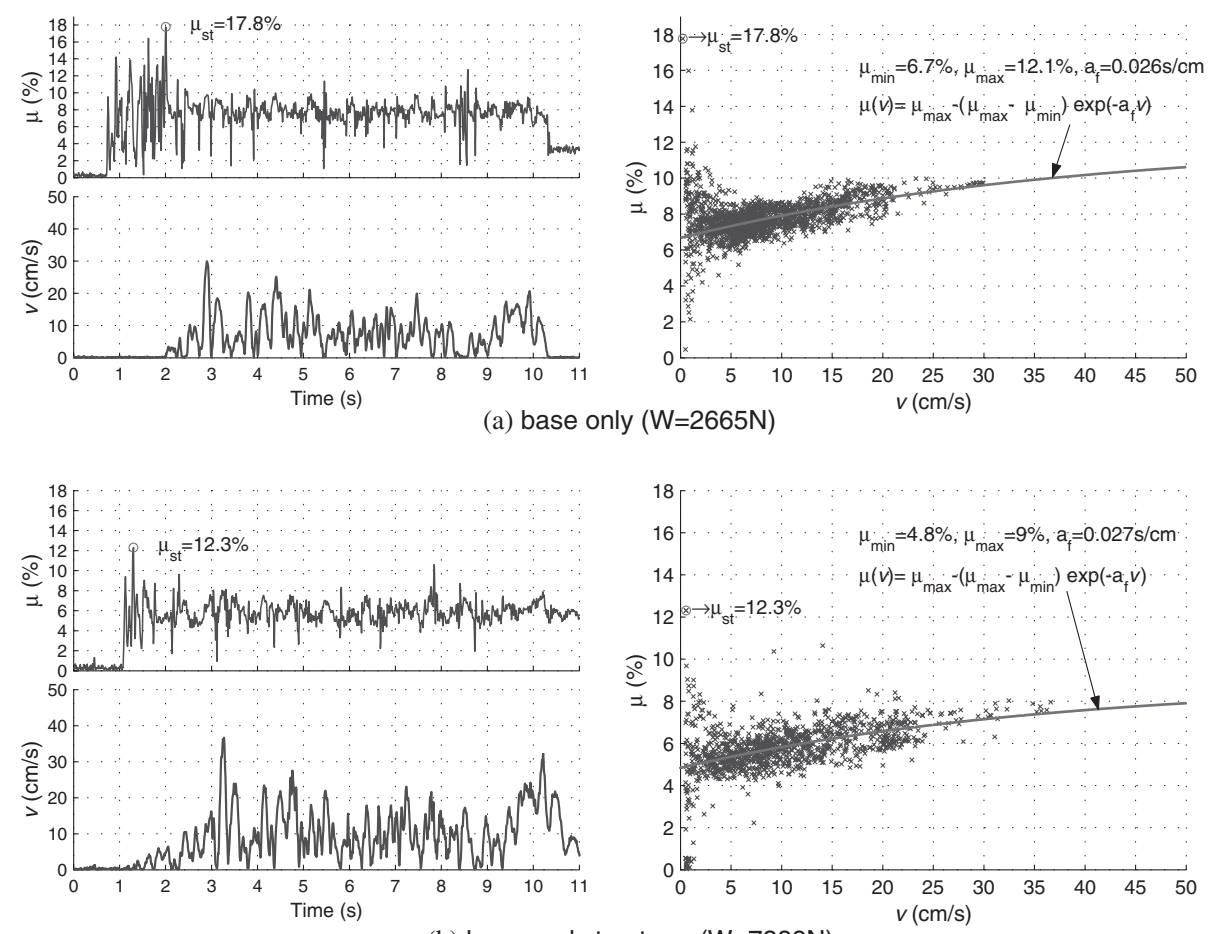

(b) base and structure $(\mathrm{W}=7389 \mathrm{~N})$

Figure 6. Identified values of the kinetic friction coefficient for the base and the base with the superstructure subject to the Synthetic \#1 record.

provide an estimate of the instantaneous value for the average frictional coefficient $\bar{\mu}$ for the 4 FPS isolators [6].

The analytical expression proposed by Constantinou et al. [11] is then used to fit the response history of the average friction coefficient $\bar{\mu}$

$$
\bar{\mu}(\bar{v})=\mu_{\max }-\left(\mu_{\max }-\mu_{\min }\right) \exp \left(-a_{\mathrm{f}} \bar{v}\right)
$$

where $\bar{v}=\left(\sum_{k=1}^{4}\left\|\dot{\boldsymbol{\delta}}_{k}\right\|\right) / 4$ is the average speed of the 4-FPS isolators; with $\dot{\boldsymbol{\delta}}_{k}=\left[\dot{\delta}_{k x}, \dot{\delta}_{k y}, \dot{\delta}_{k z}\right]^{\mathrm{T}}$ determined by numerical differentiation of the isolator deformation histories $\boldsymbol{\delta}_{k}$ measured during the test; $\mu_{\min }$ and $\mu_{\max }$ are frictional coefficients at zero and maximum speed; and $a_{\mathrm{f}}$ is a transition coefficient.

A typical result obtained from applying this identification procedure to the measured responses of the symmetric base subject to the Synthetic\#1 record selected are presented in part (a) of Figure 6. Shown on the left column of plots are the histories of friction coefficient $\bar{\mu}$ (above) and the average speed of the 4 FPS isolators $\bar{v}$ (below). These two results are combined at each instant in the plot shown at the right column to enable us to evaluate the dependency of $\bar{\mu}$ with the isolator deformation speed. The solid line presented in this plot corresponds to the theoretical estimate, which coefficients were obtained by a non-linear regression of the experimental data [6]. It is apparent that the theoretical estimate predicts 


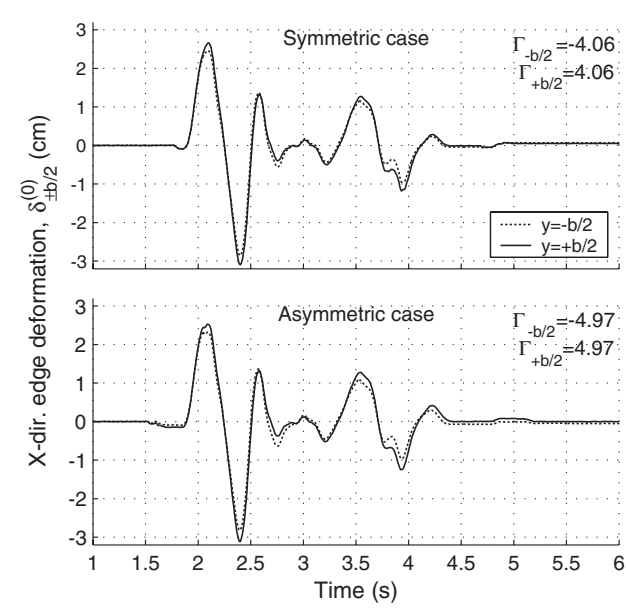

(a) Newhall

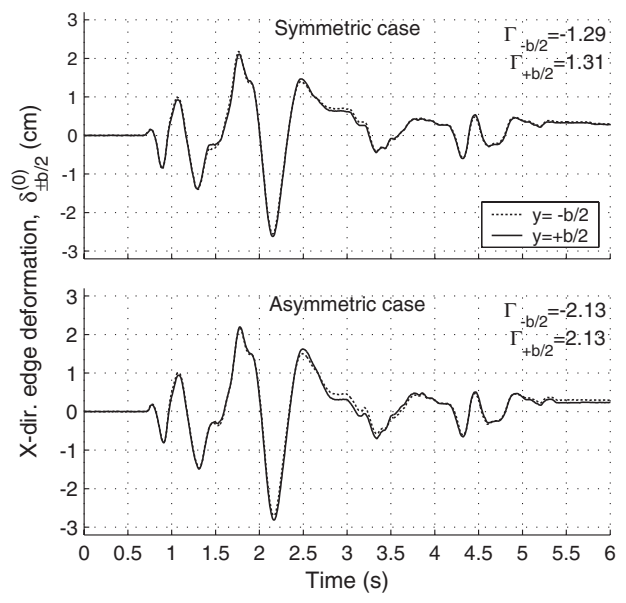

(b) $2 \times$ El Centro

Figure 7. Comparison between the measured responses at the edge of the base with symmetric and asymmetric mass distribution subject to (a) Newhall and (b) $2 \times$ El Centro record.

well the trends observed; however, the dispersion shown by the experimental data around this trend insinuates that in this case the frictional phenomena is more complex than predicted by Equation (7). The identified values for $\mu_{\min }$ vary between 6 and $7 \%$, and those for $\mu_{\max }$ between 11 and $13 \%$. Besides, the effect of static friction is apparent when sliding starts, leading to static frictional coefficient values $\mu_{\mathrm{st}} \approx 17.8 \%$. Notice that when deformation speeds are small, the dynamic friction coefficient drops at about half of its value for strong motion. It can be shown that the variation of these coefficients among different records is usually less than $20 \%[6]$.

Shown in Figure 7 are the response histories for the symmetric and asymmetric cases of the isolated base subject to the three components of the Newhall and $2 \times \mathrm{El}$ Centro record. The solid and dashed lines represent the $X$-direction deformation of the edges defined by $y= \pm b / 2$, respectively. In spite of the significant mass eccentricity, both edge deformations are very similar, showing that the FPS is capable of controlling the asymmetry caused by the eccentricity in mass. Although small in value, larger discrepancies consistently occur for the asymmetric case and the Newhall record. Indeed, since the dashed line in the asymmetric case for the Newhall record is always below the solid line, the edge $y=-b / 2$ behaves always as a stiff edge; a similar observation is obtained for the $2 \times$ El Centro record.

Torsional amplification values for the edge deformations are presented in Table I for the five records considered. The maximum torsional amplification observed for the symmetric case was $\Gamma_{b / 2}=4.4 \%$ with an average of $2.61 \%$ for all records; the corresponding maximum amplification for the asymmetric case was $\Gamma_{b / 2}=5 \%$ approximately with an average increase of $2.26 \%$. Therefore, deformation amplifications in the symmetric and asymmetric cases are very similar, validating the self-centring action of the FPS in structures with mass asymmetry.

Next, we interpret the frictional behaviour of the structure in the base-shear and torque space by using the concept of the ultimate storey shear and torque surfaces USST $[12,13]$. Although not explained herein, the basic properties of the USST can be effectively used to 


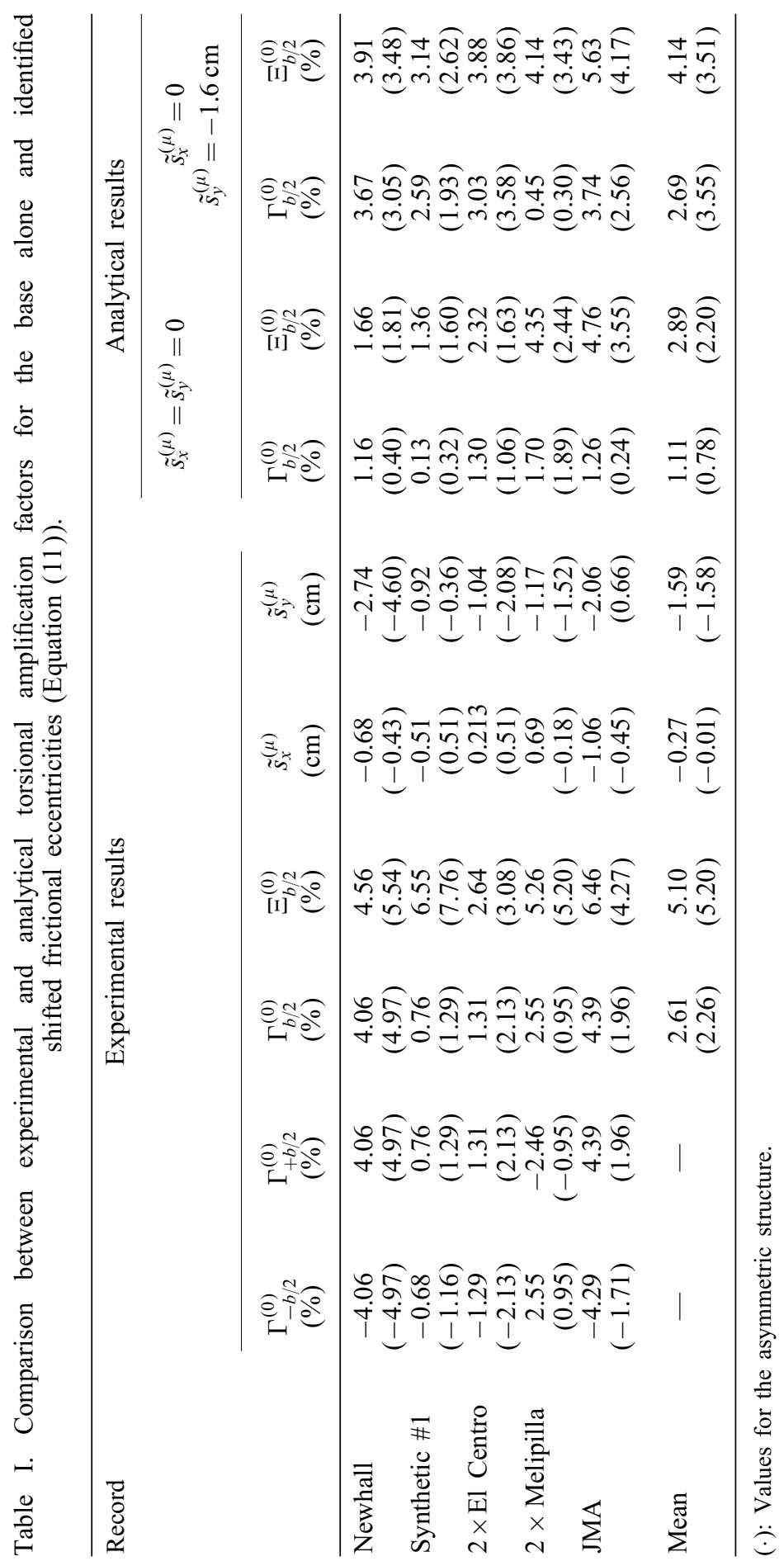


explain the complex inelastic torsional behaviour due to frictional asymmetry. Since friction is involved, these ultimate surfaces will be denoted hereafter as FUSST. Each point defining the FUSST represents a storey shear and torque combination $\mathbf{Q}_{b}^{(\mu)}=\left[Q_{x}^{(\mu)}, Q_{y}^{(\mu)}, Q_{\theta}^{(\mu)}\right]^{\mathrm{T}}$ that applied statically produces sliding of the structure. Just for convenience we normalize this force vector as follows:

$$
\hat{\mathbf{Q}}_{b}^{(\mu)}=\left[\hat{Q}_{x}^{(\mu)}, \quad \hat{Q}_{y}^{(\mu)}, \quad \hat{Q}_{\theta}^{(\mu)}\right]^{\mathrm{T}}=\left[\begin{array}{lll}
\frac{Q_{x}^{(\mu)}}{C_{n}^{(\mu)}}, & \frac{Q_{y}^{(\mu)}}{C_{n}^{(\mu)}}, & \frac{Q_{\theta}^{(\mu)}}{C_{n}^{(\mu)} \bar{\rho}_{\mu}}
\end{array}\right]^{\mathrm{T}}
$$

where $C_{n}^{(\mu)}=\mu_{n} W_{\mathrm{T}}$ is the nominal frictional capacity of the isolation system and $\mu_{n}$ is the nominal friction coefficient; $W_{\mathrm{T}}$ is the weight of the structure; and $\bar{\rho}_{\mu}=\left(\sum \mu_{k} \bar{N}_{k}\left(x_{k}^{2}+\right.\right.$ $\left.\left.y_{k}^{2}\right) / \sum \mu_{k} \bar{N}_{k}\right)^{1 / 2}$ is the nominal radius of gyration of the frictional resistance relative to the geometric center (GC) of the plan, where $\bar{N}_{k}$ is the static normal load on the $k$ th isolator.

An identification of the FUSST requires that the system reaches a large number of different shear and torque combinations on this surface. It so happens that the best excitation to identify the FUSST is a combination of uncorrelated pseudo white-noises in translation about the Xand Y-direction and rotation about the vertical $Z$-axis; combining lateral and torsional inputs is one of the advantages of the three-dimensional simulator used. The motion generated was denoted as $\mathrm{WN} / \mathrm{T} \& \mathrm{R} / 1 \mathrm{~cm} / 1 \div 70 \mathrm{rad}$, which means a white-noise signal (WN) in translation and rotation $(T \& R)$ with maximum translation amplitude of $1 \mathrm{~cm}$ and a rotation of $1 / 70$ radians. Before proceeding, the reader should be aware, however, that since frictional forces depend on the values of the normal forces in the isolators, which vary continuously in time, the limits of the FUSST in the shear and torque space may not be sharply defined.

The response histories of the symmetric and asymmetric isolated base are presented in Figure 8. Just for the sake of brevity, only the responses for the input applied in the $X-$ $\Theta$ direction are presented herein; the $\mathrm{Y}-\Theta$ results may be found elsewhere [6]. The upper row of plots shows the normalized shear and torque combinations sampled at $200 \mathrm{~Hz}$ for the isolated-base in stick or slip condition and for the symmetric (left) and asymmetric (right) configurations. Furthermore, the lower row of plots shows only the shear and torque combinations that cause slipping of the isolated base, sampled at $100 \mathrm{~Hz}$. In the latter, the normalized deformation velocities $\dot{\mathbf{q}}_{n}=\left[\dot{q}_{x}, \dot{q}_{y}, \dot{q}_{\theta} \bar{\rho}_{\mu}\right]^{\mathrm{T}}$, computed by numerical differentiation of the deformations obtained during the test, are identified by arrows with length proportional to their magnitude, $\left\|\dot{\mathbf{q}}_{n}\right\|$. As a reference, a section of the nominal FUSST, defined by the plane $Q_{y n}^{(\mu)}=0$ and a nominal friction coefficient $\mu_{n}=0.08$, is shown by dashed lines in the figure. Conventionally, the torques are referred to the GC of the plan; thus, the section of the FUSST is symmetric and asymmetric in the $X-\Theta$ plane for the symmetric and asymmetric mass configurations, respectively. Several interesting observations can be stated related to this figure.

First, it is apparent that in both cases the storey shears and torque combinations tend to concentrate around the nominal FUSST. This is important since it validates experimentally the concept of the FUSST, which has also been proposed for simplified three-dimensional inelastic analysis of multistorey buildings and experimentally validated with the recorded accelerations in a real structure [14]. It is apparent, however, that some shear and torque combinations go beyond the FUSST as a result of two main causes: isolator sticking and variation of the friction coefficient $\mu$ with deformation velocity. Indeed, notice in the lower plots that the 

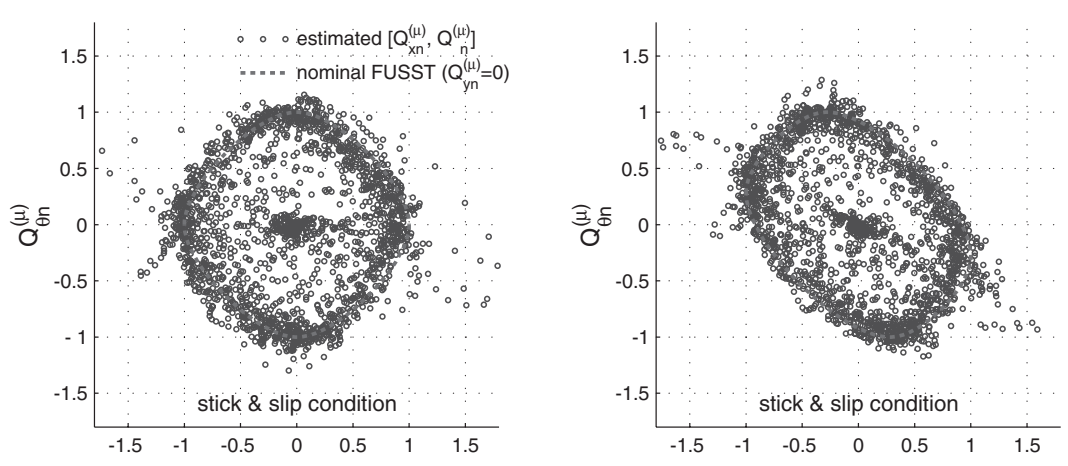

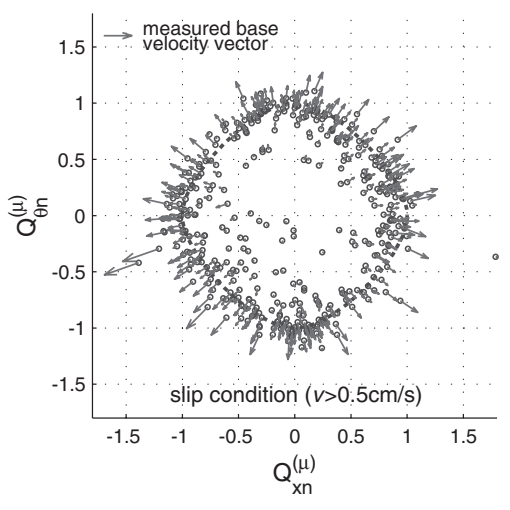

(a) symmetric structure

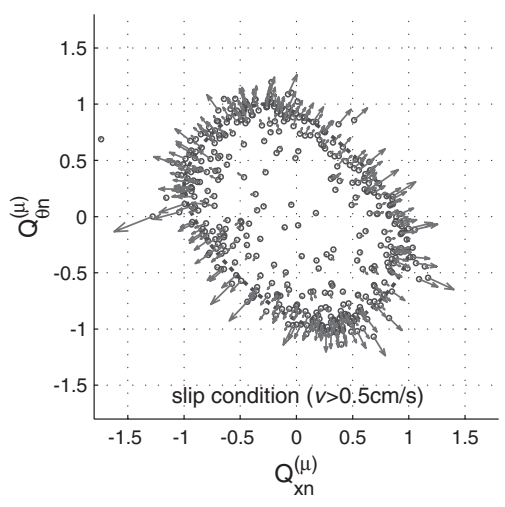

(b) asymmetric structure

Figure 8. Experimental identification of the FUSST for the isolated base with symmetric and asymmetric mass distribution, subject to the pseudo white-noise ground motion $\mathrm{WN} / \mathrm{T} \& \mathrm{R} / 1 \mathrm{~cm} / 1 \div 70 \mathrm{rad}$.

larger arrows correspond to points that lie outside of the FUSST, implying that the friction coefficient increases with larger deformation velocities. On the other hand, the two points that lie farther from the origin of the shear and torque space are points with zero velocity for which sticking occurs. Another interesting observation is that the vectors of deformation velocity are all orthogonal to the FUSST, validating the well known associated-flow rule commonly used in perfect plasticity, which states that the plastic deformation increment occurs in a direction perpendicular to the FUSST. This rule is also confirmed by looking at the points inside the FUSST which have essentially a zero velocity. Consequently, it seems natural to use the idea of a macro-element model $[12,13]$ for the analysis and design of frictional isolation systems such as the FPS.

Results presented in Figure 8 can be used further to identify the inelastic parameters of the system. For instance, it can be shown [6] that the locus of all shear combinations that lead to purely translational mechanisms, in which $\dot{q}_{\theta}=0$, may be represented by a planar surface $\Pi_{t p}$ defined by

$$
\Pi_{t p}\left(Q_{x n}^{(\mu)}, Q_{y n}^{(\mu)}, Q_{\theta n}^{(\mu)}\right)=\frac{-e_{y}^{(\mu)}}{\bar{\rho}_{\mu}} Q_{x n}^{(\mu)}+\frac{e_{x}^{(\mu)}}{\bar{\rho}_{\mu}} Q_{y n}^{(\mu)}-Q_{\theta n}^{(\mu)}=0
$$



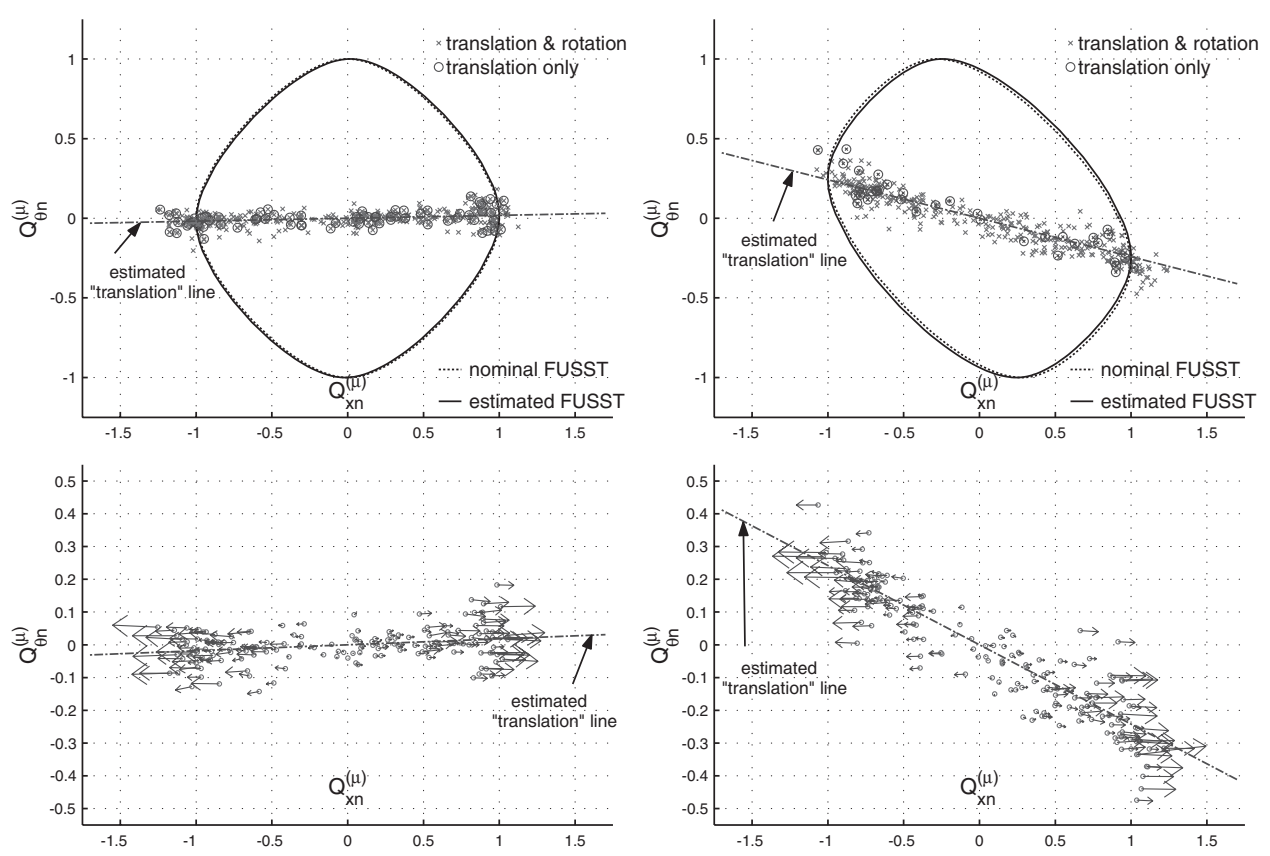

(a) symmetric structure

(b) asymmetric structure

Figure 9. Evaluation of the frictional eccentricities of the symmetric and asymmetric base by using the 'plane of pure translation' and the $2 \times$ El Centro record.

where $e_{x}^{(\mu)}$ and $e_{y}^{(\mu)}$ are the frictional eccentricities of the system. Equation (10) may be used to estimate the frictional eccentricities by the following procedure: (i) identify instants $t^{*}$ for which $\dot{q}_{\theta}$ is zero; (ii) store the shear and torque vector $\mathbf{Q}_{b}^{(\mu)}\left(t^{*}\right)=\left[Q_{x n}^{(\mu)}\left(t^{*}\right), Q_{y n}^{(\mu)}\left(t^{*}\right), Q_{\theta n}^{(\mu)}\left(t^{*}\right)\right]^{\mathrm{T}}$ at those instants of pure translation; (iii) perform a linear regression to fit a plane through the sequence $\mathbf{Q}_{b}^{(\mu)}\left(t^{*}\right)$ by using Equation (9) as a functional, i.e.

$$
\left[\tilde{e}_{x}^{(\mu)}, \tilde{e}_{y}^{(\mu)}\right]=\min _{e_{x}^{(\mu)}, e_{y}^{(\mu)}} \sum_{k}\left(\frac{-e_{y}^{(\mu)}}{\bar{\rho}_{\mu}} Q_{x n}^{(\mu)}\left(t_{k}^{*}\right)+\frac{e_{x}^{(\mu)}}{\bar{\rho}_{\mu}} Q_{y n}^{(\mu)}\left(t_{k}^{*}\right)-Q_{\theta n}^{(\mu)}\left(t_{k}^{*}\right)\right)^{2}
$$

where $\tilde{e}_{x}^{(\mu)}$ and $\tilde{e}_{y}^{(\mu)}$ represent the estimated or identified frictional eccentricities. For later convenience, the identified frictional eccentricities are shifted with respect to their nominal value, i.e.

$$
\tilde{s}_{x}^{(\mu)}=\tilde{e}_{x}^{(\mu)}-\bar{e}_{x}^{(\mu)} ; \quad \tilde{s}_{y}^{(\mu)}=\tilde{e}_{y}^{(\mu)}-\bar{e}_{y}^{(\mu)}
$$

where $\bar{e}_{x}^{(\mu)}$ and $\bar{e}_{y}^{(\mu)}$ represent the frictional eccentricities associated with the nominal frictional capacities, i.e. the nominal coordinates of the center of frictional resistance (CF), measured with respect to the GC of the plan.

The procedure just described to compute the frictional eccentricities is shown in Figure 9 for the symmetric (left column) and asymmetric (right column) configurations subject to the 
$2 \times$ El Centro record. The 'measured' shear and torque combinations $\left(Q_{x n}^{(\mu)}, Q_{\theta n}^{(\mu)}\right)$ are identified by the small crosses, while the circles indicate points in which pure translation occurs. The nominal and estimated FUSST are presented by dashed and solid lines, respectively; the trace of the identified plane $\Pi_{t p}$ in the $Q_{x n}^{(\mu)}-Q_{\theta n}^{(\mu)}$ plane is also presented. The estimated FUSST is computed by using different coefficients of friction for the different FPS, leading to the objective frictional eccentricities $\tilde{e}_{x}^{(\mu)}$ and $\tilde{e}_{y}^{(\mu)}$. Furthermore, in the lower row of plots the base shear and torque is presented with arrows proportional to the normalized deformation velocities $\dot{\mathbf{q}}_{n}$. It is apparent how positive and negative torsional deformation velocities tend to lie, as it should, above and below plane $\Pi_{t p}$, respectively. The estimated shift of the frictional eccentricities for the different earthquake motions and analysis direction are summarized in columns 5 and 6 of Table I. Notice that $\tilde{e}_{x}^{(\mu)}$ is usually around zero, its nominal value; instead, $\tilde{e}_{y}^{(\mu)}$ has a strong tendency to be smaller than its nominal value. It is interesting to see that there is no strong difference between the position of the CF in the symmetric and asymmetric case, implying that the FPS is capable of naturally controlling mass asymmetry in the superstructure.

Before looking into the behaviour of the base and superstructure together, the earthquake results obtained from these experiments are compared with the analytical predictions. The analytical model of the base considers as input the true six components of acceleration 'measured' at the base. Moreover, the FPS are represented by a physical model developed recently $[5,6]$, including large deformations, sticking, and the velocity-dependent values of the friction coefficient $\tilde{\mu}$ identified for each particular test (Figure 6). Furthermore, to include the identified frictional asymmetry in plan, the nominal position of the CF is shifted by a mean value $\tilde{s}_{y}^{(\mu)}=-1.6 \mathrm{~cm}$ for the five records only in $Y$-direction (Table I), for the symmetric and asymmetric configurations. To achieve this frictional eccentricity, the frictional coefficients of isolators 1 and 2 were increased by $5 \%$, while the coefficient for isolators 3 and 4 were reduced $5 \%$.

Shown in Figure 10 is a comparison between the experimental and analytical results for both structural configurations subject to the Newhall record. Similar results are obtained for all other records [6]. The plots on the left show the response histories of the base displacements in the $X, Y$, and $\Theta$-directions, respectively. On the other hand, the plots on the right show the response histories of the normalized base shear and torque. The agreement between the experimental and analytical results in terms of the lateral deformations and base shear is exceptional. This is due primarily to the accuracy of the large deformation model used, and the model considered to account for the variation of the friction coefficient. Although, the results in terms of rotations and torques are not as good as those in translation, the trends of the response are well predicted by the analytical model. Discrepancies in this latter case are attributed primarily to noise in the experimental measures, the variability of the frictional eccentricity assumed constant in the analytical model, and other asymmetry factors not accounted for in the analytical model. In spite of these discrepancies, and considering the complexity of the FPS behaviour, the analytical model is capable of predicting the response of the base with excellent accuracy if used with the appropriate values for the FPS parameters. Indeed, summarized in Table II are the torsional amplifications represented by the parameters $\Gamma_{b / 2}^{(0)}$ and $\Xi_{b / 2}^{(0)}$ obtained analytically; the analytical estimates include the estimated frictional eccentricity. It is apparent that ignoring this frictional eccentricity leads to rather poor estimates of the torsional amplifications. In general, analytical estimates of the torsional amplifications for the 


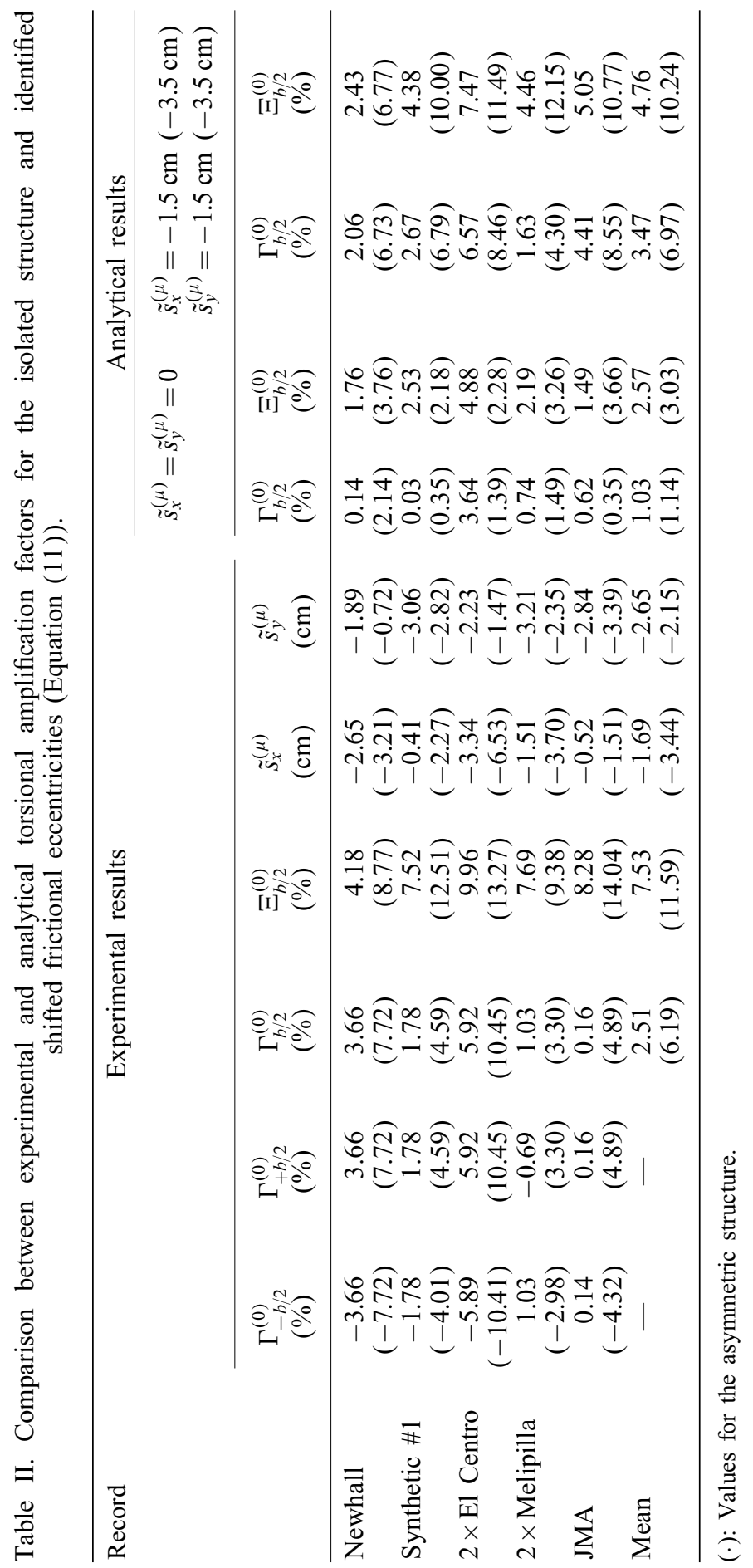



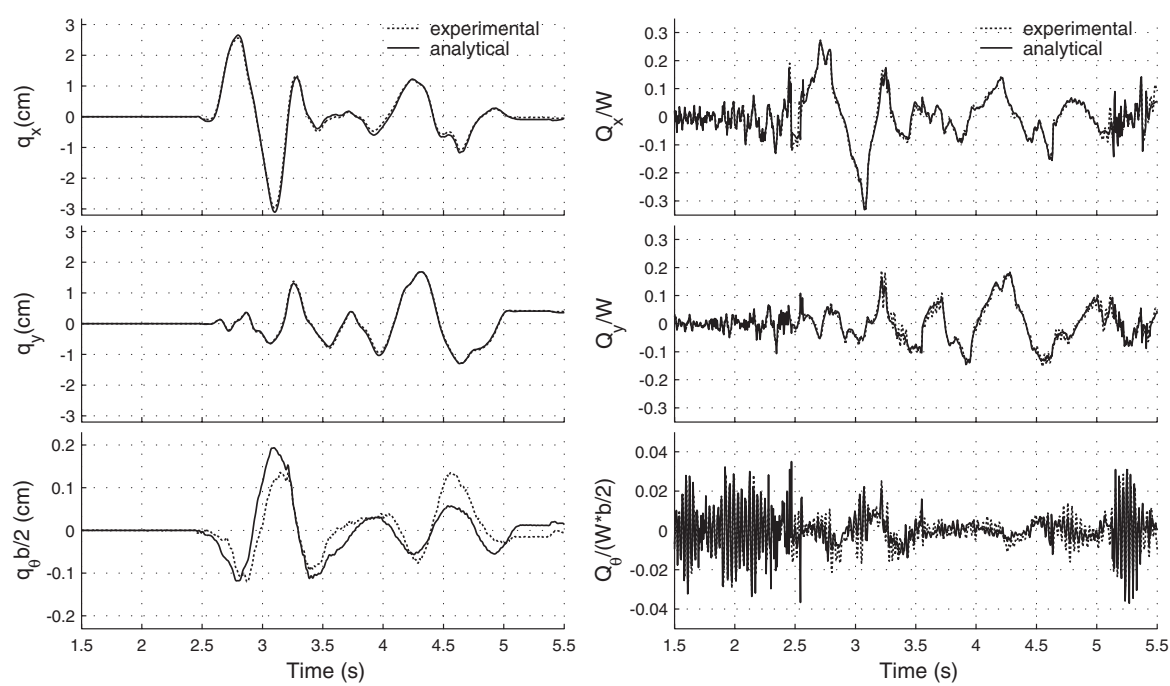

(a) Symmetric
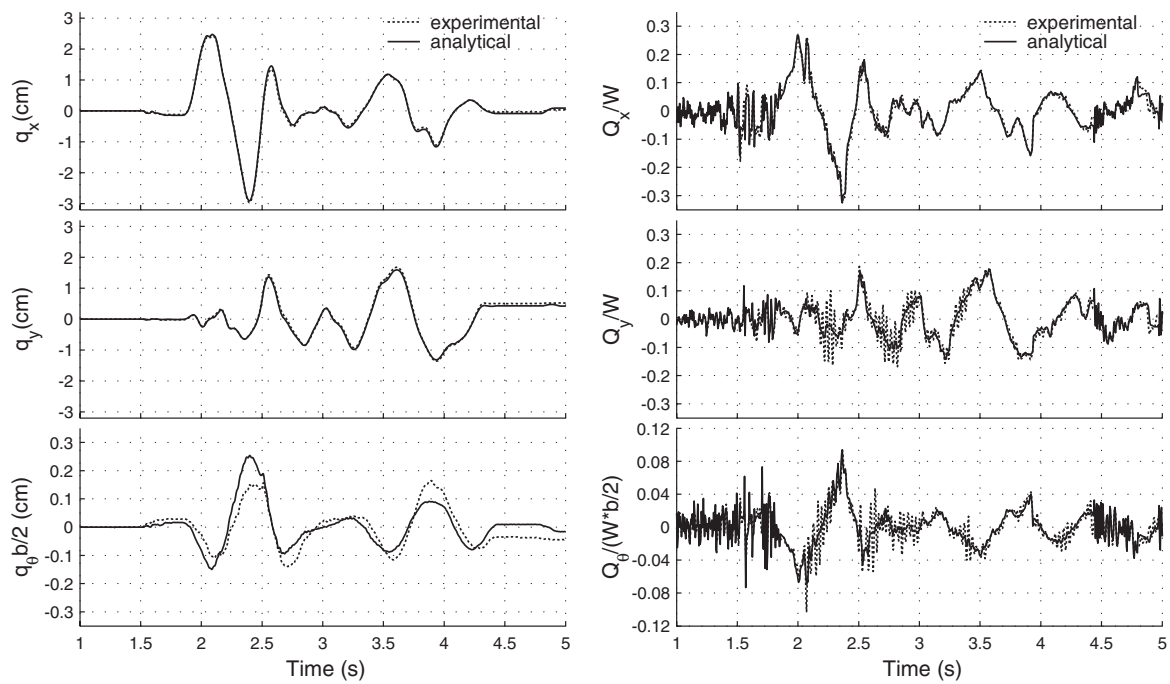

(b) Asymmetric

Figure 10. Comparison between the experimental and analytical response histories for the symmetric and asymmetric base subject to the Newhall record.

base are usually less than $5 \%$, which are of the same order as those obtained experimentally (Table I).

Finally, shown in Figure 11 is a comparison between the theoretical and experimental restoring force-deformation loops in the $X$-direction for the symmetric configuration subject to the Newhall and Synthetic \#1 records; these results carry over to the asymmetric configuration and other input motions as well [6]. The plots on the left column correspond to the input 

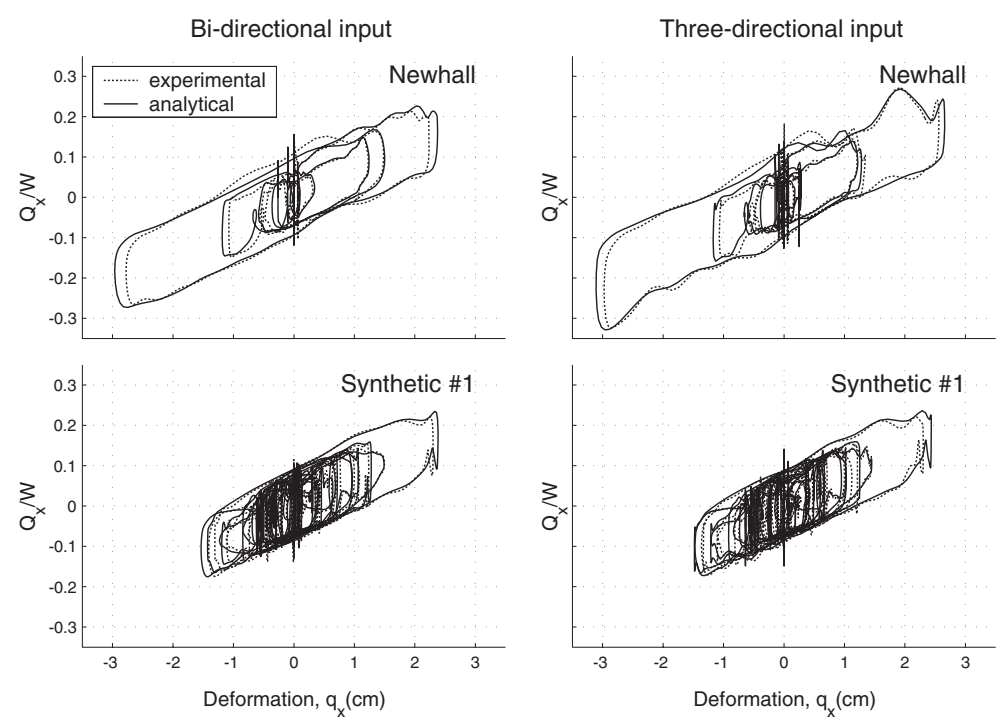

Figure 11. Comparison between the analytical and experimental force deformation loops for the symmetric configuration of the base and subject to the two horizontal and the three components of the Newhall and Synthetic \#1 ground motions.

with two horizontal components, while the plots on the right column correspond to the same horizontal input but including also the vertical component. The similarity between the analytical estimates and the experimental results are very good for both excitations. The reader may notice for the Newhall record, the significant increment in the restoring force due to the increase in normal isolator forces produced by the vertical ground-motion component. By using the experimental values, this shear increase is as large as $20 \%$ in the $X$-direction. This value coincides well with analytically predicted amplification values presented earlier [15], which were shown to be proportional to the statistical correlation between the horizontal and vertical components of the ground motion. For the Newhall record, the statistical correlation between the $X$ and $Z$-components is -0.18 . Such correlation, however, is just 0.04 for the Synthetic \#1 ground motion case and, hence, the smaller variation of the restoring force with the vertical ground motion component in the latter case. Notice also in these force-deformation loops the spikes that occur as a consequence of the sticking phenomena in the isolators.

\section{SEISMIC BEHAVIOUR OF THE THREE-STOREY STRUCTURAL MODEL}

In this section, the experimental earthquake responses of the building model are compared with the analytical predictions obtained by using a calibrated structural model of the building and isolation system. The structural model was developed using the dynamic analysis toolbox SatLab [16] that works under the MATLAB environment [17]. A rigid in-plane floor diaphragm assumption, with the conventional three degrees-of-freedom per floor level, twelve degree-offreedom per joint, and consistent masses were considered in the structural model. Also, the six components of the motion of the shaking table were used as input for the structural model. 


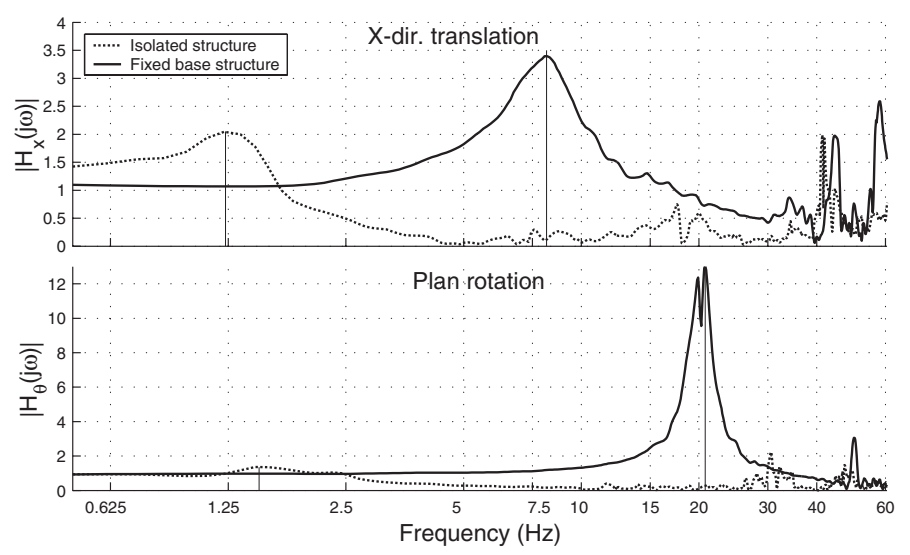

Figure 12. Empirical transfer functions (ETF) between the total accelerations of the ground and roof in the $X$ - and $\Theta$-directions for the symmetric model with fixed and isolated base.

The stiffness matrix of the superstructure was calibrated by using its fixed-base vibration periods. As an example, shown in Figure 12 are the magnitudes of the empirical transfer functions (ETF) of the fixed-base model in the $X$ and $\Theta$-directions; these results were obtained from unidirectional pseudo-white noise inputs. It is apparent that only the first translational and rotational vibration periods in each direction can be clearly identified. The identified periods for the fixed-base buildings were $0.125 \mathrm{~s}$, and $0.05 \mathrm{~s}$, for the $X$ - and $\Theta$-directions, respectively. The non-classical damping matrix of the structure was constructed based on damping ratios for the superstructure of $20 \%$ in the first and second lateral vibration modes, $6 \%$ for the third torsional mode, and 3\% for all other modes, as it was identified from the fixed-base ETF. The large damping ratio for the lateral modes is due to unforeseen non-linearities of the model, in particular, the bolted beam-column joints. Since for a torsional motion the dissipation tends to concentrate mostly along the perimeter frames, the identified damping ratios tend to be smaller in that case. Furthermore, the FPS isolators were represented by a large-deformation physical model $[5,6]$ which accounts for uplift, sticking, and the velocity dependence of the sliding coefficient. The final structural model used for analytical predictions also included the experimentally identified frictional eccentricities.

Figure 12 also shows the ETF associated with the base isolated symmetric model subject to the input signal denoted earlier as $\mathrm{WN} / \mathrm{T} \& \mathrm{R} / 1 \mathrm{~cm} / 1 \div 70 \mathrm{rad}$ that combines translation and rotation of the platform. By comparing these results with those of the fixed-base model, it can be observed a clear shift in period and increase in damping ratio as a result of the FPS system. Indeed, the fundamental isolated frequency in translation in the $X$-direction is $0.75 \mathrm{~s}$, which coincides well with the nominal value corresponding to a radius of curvature of the FPS devices, $R_{0}=14.3 \mathrm{~cm}$. Analogously, the first torsional period for the isolated structure is $0.63 \mathrm{~s}$, i.e. $\Omega_{b}=1.2$, which is also a close estimate for the nominal value of the frequency ratio. Damping ratios are not stated since they will be automatically included into the model by the constitutive relationship of the FPS isolators.

The identification of the sliding friction coefficient of the symmetric structure subject to the Synthetic\#1 record is presented in part (b) of Figure 6. It is apparent that the friction coefficient for the structure is about $40 \%$ smaller than the corresponding coefficient for the 


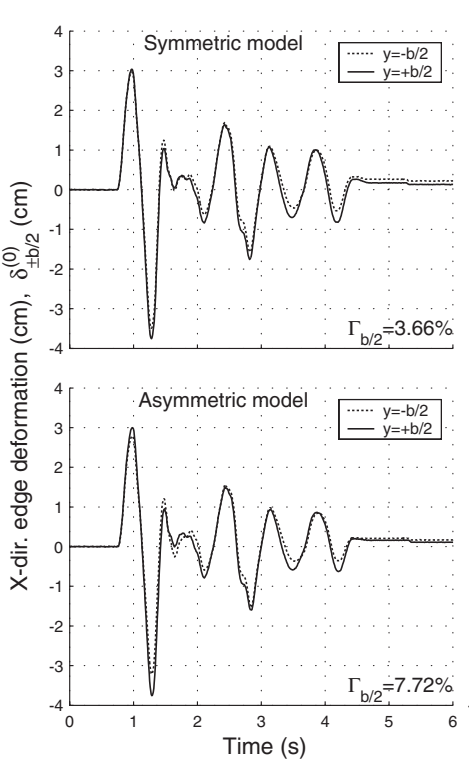

(a) Newhall

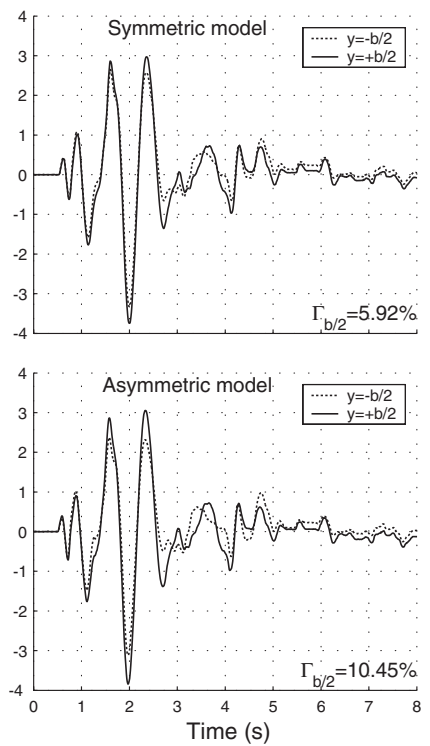

(b) $2 \times$ El Centro

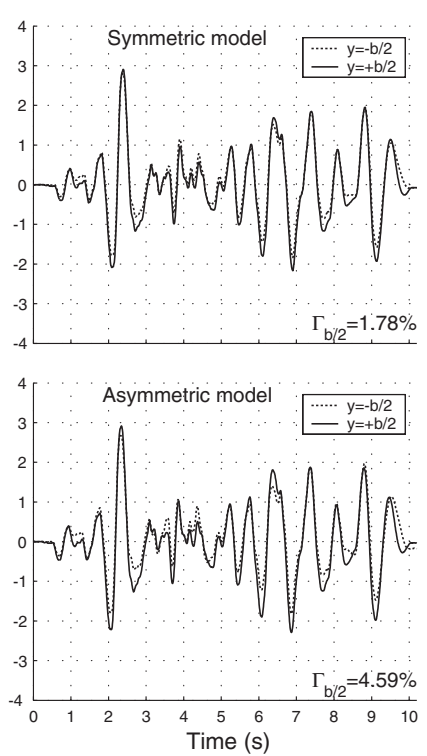

(c) Synthetic \#1

Figure 13. Experimental responses at the edge of the building for the symmetric (above) and asymmetric (below) models, subject to the Newhall, $2 \times$ El Centro, and Synthetic \#1 ground motions.

base alone. Such reduction in friction is the result of the higher contact pressure on the sliding surface [11], leading to friction coefficients for the structure that vary between 4.8 and $9 \%$.

Described next are the earthquake responses of the symmetric and asymmetric plan configurations of the three-storey model subject to the family of five ground motions considered. Recall that the asymmetric building case has a mass eccentricity $e_{y} / b=0.14 b$ in the positive $Y$-direction. Shown in Figure 13 are the $X$-direction edge deformation histories at the base $(y= \pm b / 2)$ of the building due to the Newhall, $2 \times$ El Centro, and Synthetic \#1 ground motions. By comparing these results with the same deformation histories for the isolated base presented in Figure 7, it is observed that both have similar frequency content and follow similar trends, although these responses at the base are about $30 \%$ larger than those of the base alone. This is a consequence of the decrease in the sliding coefficient due to the almost $300 \%$ increase in contact pressure developed when the superstructure is present. Also, the torsional amplifications for the building model vary from 1.8 to $5.9 \%$ and from 4.6 to $10.5 \%$ for the symmetric and asymmetric configurations, respectively. Consequently, torsional amplifications at the base for the asymmetric case are essentially twice as large as the values for their symmetric counterpart.

Presented in Table II are the measured torsional amplifications at the base of the structure for the symmetric and asymmetric mass configurations in the superstructure. These results are comparable with those presented earlier in Table 1 for the base alone. It is shown for the symmetric case that the mean increase in edge deformations at the base due to accidental torsion $\Gamma_{b / 2}^{(0)}$ is $2.51 \%$, which is similar to the one computed for the base alone (Table I). Notice also that the values of the shifted frictional eccentricities (Equation (11)) are both negative, implying that rotation of the plan tends to occur with respect to a point located in the third 
quadrant of the building plan (Figure 1). The corresponding mean torsional amplification for the asymmetric case is $6.2 \%$, which is due to the larger frictional eccentricities identified for the asymmetric structure. These frictional eccentricities also correspond to a point located in the third quadrant of the building.

Figure 14 shows base deformation and force histories at the $\mathrm{CM}$ of the base analogous to the ones presented earlier for the base alone in Figure 10. Results for other earthquakes lead to similar conclusions and are omitted here for brevity [6]. As stated before, it is clear that the structural model is capable of reproducing accurately the translational motion of the base in spite of the presence of the superstructure. Analytical errors are similar for both structural configurations and the asymmetry in the superstructure does not impact the accuracy of the model. By including the frictional eccentricity concept in the model, even the small torsional behaviour can be predicted satisfactorily. Although expected, this is a remarkable attribute of base isolated structures: 'the uncertainty of the model predictions are small and do not vary significantly with the presence and characteristics of the superstructure'. Such argument does not seem to have impacted the profession, as it should, so far. In the opinion of the authors, the large uncertainty present in conventional structures [18] does not allow to achieve precise performance objectives as it does by using base isolation.

A summary of the predicted torsional amplifications at the base of the symmetric and asymmetric structures are presented in columns 7-10 of Table II. Just to stress the importance of the value chosen for the frictional eccentricity, columns 7 and 8 show the values of $\Gamma_{b / 2}^{(0)}$ and $\Xi_{b / 2}^{(0)}$ associated with the nominal value of frictional eccentricity, i.e. $\tilde{s}_{x}^{(\mu)}=\tilde{s}_{y}^{(\mu)}=0$. By comparing columns 7 and 8 with columns 3 and 4, it is observed that the resulting discrepancies between the actual and predicted torsional amplifications are important in both cases. Consequently, columns 9 and 10 summarize the values for $\Gamma_{b / 2}^{(0)}$ and $\Xi_{b / 2}^{(0)}$ obtained by increasing artificially the nominal frictional eccentricities in $0.011 b=1.5 \mathrm{~cm}$ (symmetric case) and $0.025 b=3.5 \mathrm{~cm}$ (asymmetric case). The mean values of these analytical predictions vary from 3.5 to $4.8 \%$ and from 7 to $10.2 \%$ for the symmetric and asymmetric cases, respectively, which turn out to be similar to the experimental values provided in columns 3 and 4 of Table II.

As stated in previous work [10] accidental and natural torsion effects may be larger in the superstructure than at the base. In order to evaluate this, Figure 15 shows the normalized shear and torque histories of the superstructure associated with the symmetric and asymmetric plan configurations. Similar results with identical features are presented elsewhere for other ground motions [6]. It is apparent that storey shears are well predicted by the analytical model for both structural configurations. This is not surprising since the shear histories are controlled by the identified low frequency motion corresponding to the fundamental isolated period in translation. However, the storey torque shows larger discrepancies, especially in the symmetric configuration; storey torques are quite well represented for the superstructure with asymmetric mass distribution where rotations are more significant than in the symmetric case. The major discrepancies are related to the high frequency content of the response, attributed to contribution of higher modes in the structure, which cannot be easily identified experimentally.

Next, a comparison is presented between torsional amplifications obtained from the experimental model with fixed-base and base isolation. The objective of these comparisons is to evaluate the capacity of the FPS system to control the torsional response of the superstructure caused by accidental and natural torsion. Shown in Figure 16 are the storey shear and torque histories for the model subjected to the $2 \times$ Melipilla record. It is apparent that the reduction 

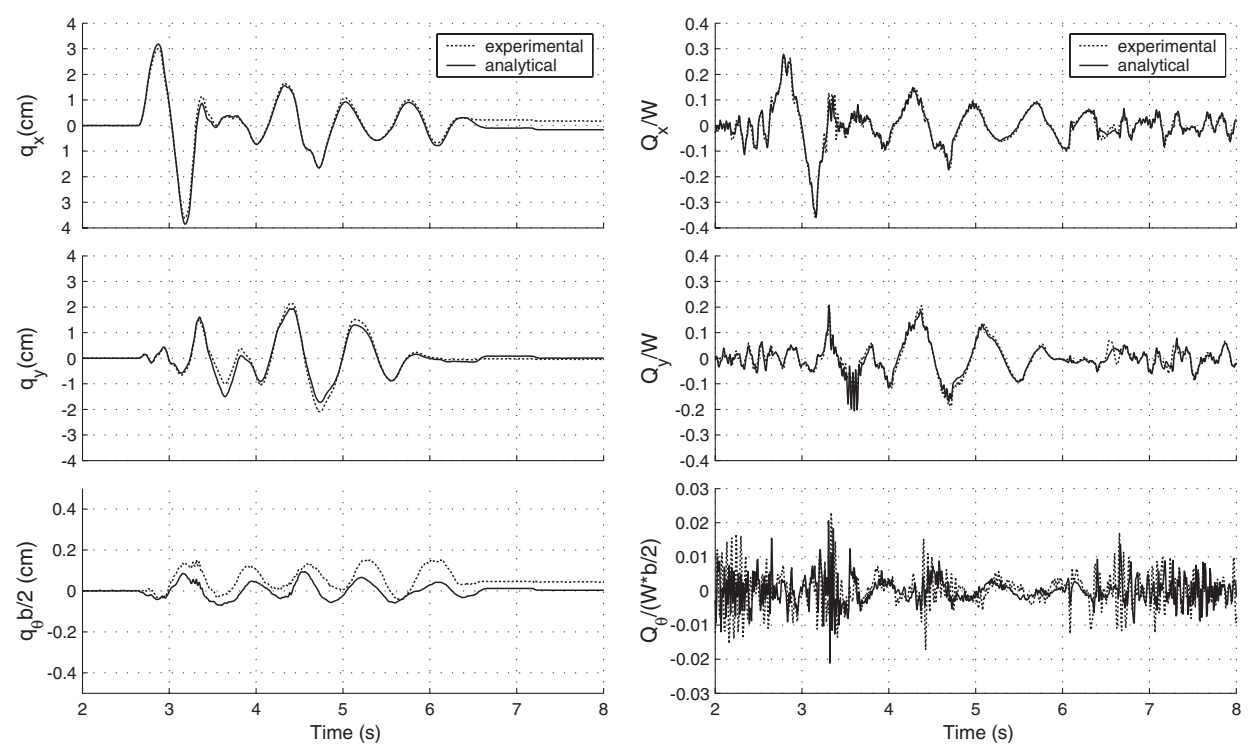

(a) symmetric structure
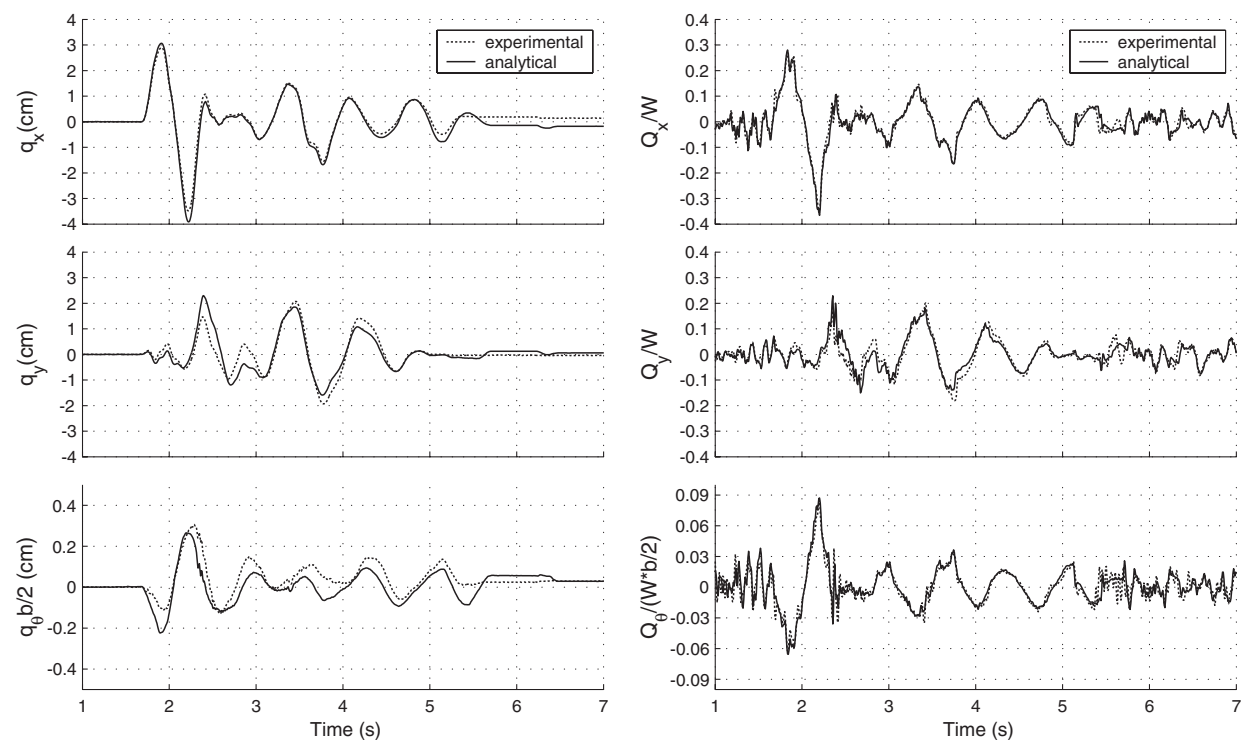

(b) asymmetric structure

Figure 14. Comparison between the analytical and experimental deformation, shear, and torque histories for the symmetric and asymmetric model subject to the Newhall record.

factors are greater in storey torque than in storey shear for both structural configurations. Reduction factors for storey shear and torque tend to be larger in the nominally symmetric case; they reach 5.3 and 13.3 for the shear and torque at the base, respectively, and vary to 4.9 and 7.2 for the asymmetric case, respectively. Reduction factors tend to be smaller at 

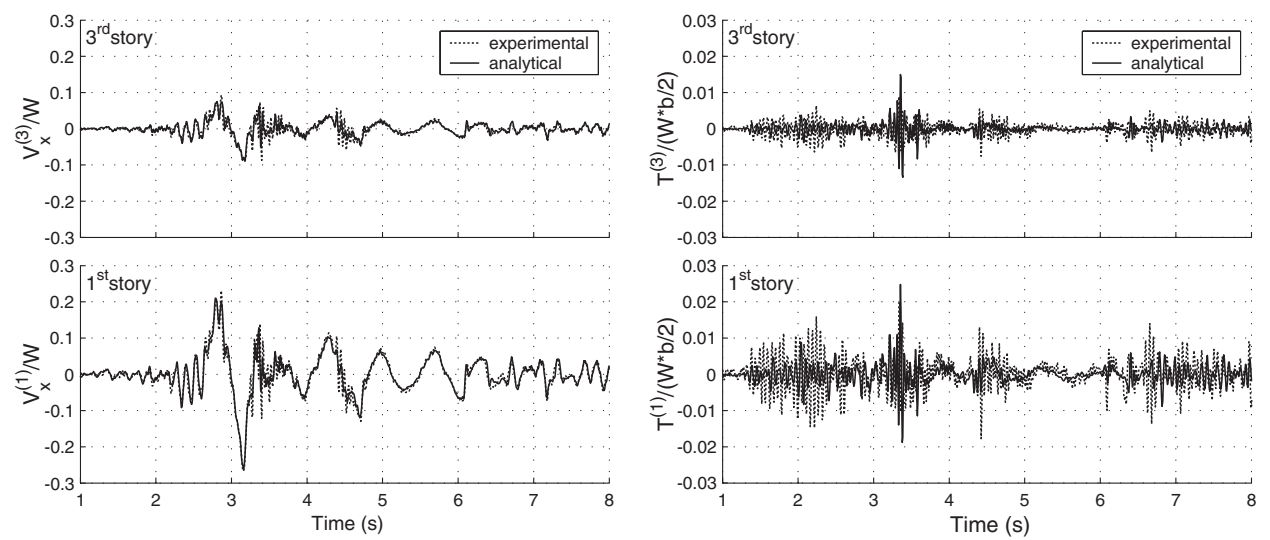

(a) symmetric structure
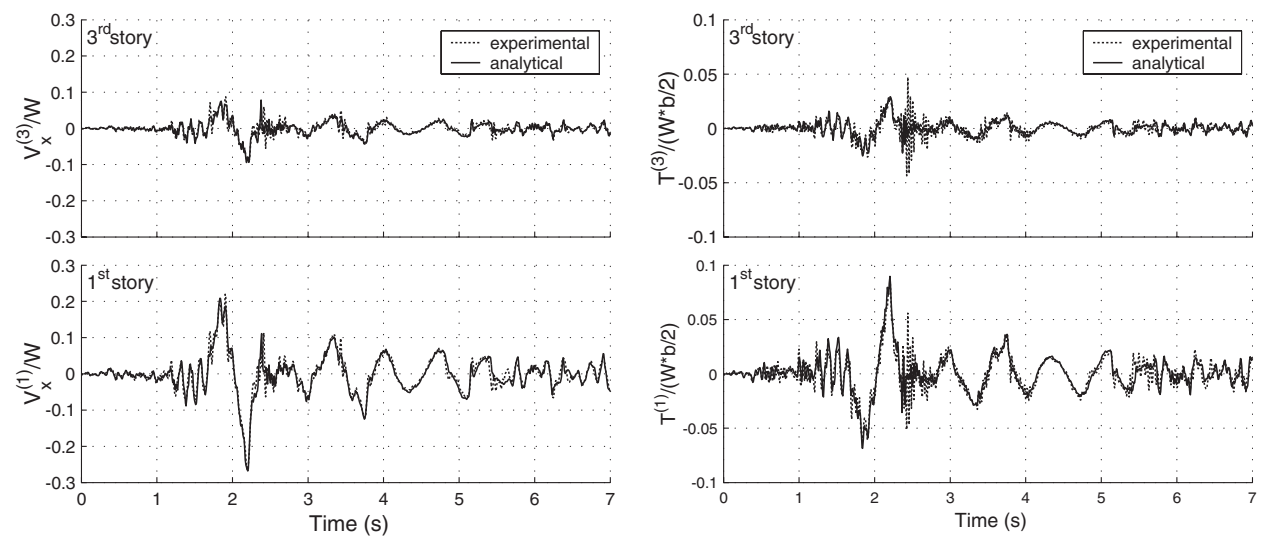

(b) asymmetric structure

Figure 15. Comparison between the experimental and analytical shear and torque histories of the superstructure with symmetric and asymmetric mass configuration subject to the three components of the Newhall record.

higher stories of the structure as a result of the larger contribution of higher modes in those stories. Higher mode contributions are especially important in structures isolated with the FPS system because of the stick-slip phenomenon present in the isolators.

Shown in Table III are the force reduction factors obtained for the nominally symmetric and asymmetric model, respectively, subject to the three ground motion components of each of the five records considered in this study. The mean shear reduction for all stories and records is about 3.9 and 4 for the two symmetric and asymmetric configurations, respectively. The corresponding values of the reduction factor for the storey torque are 7.3 and 4.8, respectively. These reduction factors, however, will not coincide with those for the forces in structural members, since peak shear and torque values do not occur simultaneously.

Because storey shear and torque have a direct relationship with the interstorey deformation through the stiffness of the storey, it is possible to propose an approximate expression to 
Table III. Shear and torque reduction factors of the symmetric and asymmetric isolated structure with respect to the fixed-base structure.

\begin{tabular}{|c|c|c|c|c|c|c|}
\hline \multirow[t]{3}{*}{ Record } & \multicolumn{6}{|c|}{ Reduction factor } \\
\hline & \multicolumn{3}{|c|}{$X$-dir. shear } & \multicolumn{3}{|c|}{ Torque } \\
\hline & Storey 1 & Storey 2 & Storey 3 & Storey 1 & Storey 2 & Storey 3 \\
\hline Newhall & 3.08 & $\begin{array}{c}3.69 \\
(364)\end{array}$ & 2.93 & $\begin{array}{c}9.55 \\
(376)\end{array}$ & 11.34 & 9.17 \\
\hline Synthetic \#1 & $\begin{array}{c}3.22 \\
(3.64)\end{array}$ & $\begin{array}{c}2.48 \\
(3.83)\end{array}$ & $\begin{array}{c}2.51 \\
(3.91)\end{array}$ & $\begin{array}{c}3.95 \\
(5.89)\end{array}$ & $\begin{array}{c}3.60 \\
(6.16)\end{array}$ & $\begin{array}{c}4.41 \\
(6.07)\end{array}$ \\
\hline $2 \times$ El Centro & $\begin{array}{c}3.13 \\
(3.37)\end{array}$ & $\begin{array}{c}3.19 \\
(3.23)\end{array}$ & $\begin{array}{c}3.26 \\
(2.94)\end{array}$ & $\begin{array}{l}6.46 \\
(2.94)\end{array}$ & $\begin{array}{c}5.96 \\
(3.27)\end{array}$ & $\begin{array}{c}5.72 \\
(3.25)\end{array}$ \\
\hline $2 \times$ Melipilla & $\begin{array}{c}5.27 \\
(4.92)\end{array}$ & $\begin{array}{c}5.00 \\
(4.10)\end{array}$ & $\begin{array}{c}4.97 \\
(3.78)\end{array}$ & $\begin{array}{l}13.27 \\
(7.20)\end{array}$ & $\begin{array}{l}11.99 \\
(5.41)\end{array}$ & $\begin{array}{c}7.92 \\
(4.47)\end{array}$ \\
\hline JMA & $\begin{array}{c}4.58 \\
(4.44)\end{array}$ & $\begin{array}{c}5.19 \\
(5.05)\end{array}$ & $\begin{array}{c}5.68 \\
(3.98)\end{array}$ & $\begin{array}{c}6.32 \\
(5.08)\end{array}$ & $\begin{array}{c}5.42 \\
(4.73)\end{array}$ & $\begin{array}{c}4.82 \\
(4.57)\end{array}$ \\
\hline Mean & $\begin{array}{c}3.86 \\
(3.87)\end{array}$ & $\begin{array}{c}3.91 \\
(3.97) \\
3.88(3.94)\end{array}$ & $\begin{array}{c}3.87 \\
(3.98)\end{array}$ & $\begin{array}{c}7.91 \\
(5.08)\end{array}$ & $\begin{array}{c}7.66 \\
(4.73) \\
7.33(4.79)\end{array}$ & $\begin{array}{c}6.41 \\
(4.57)\end{array}$ \\
\hline
\end{tabular}

$(\cdot)$ : Values for the asymmetric structure.

Table IV. Experimental torsional amplification factors (Equation (12)) of the symmetric and asymmetric structure with fixed-base and base isolation.

\begin{tabular}{|c|c|c|c|c|c|c|}
\hline \multirow[t]{3}{*}{ Record } & \multicolumn{6}{|c|}{ Torsional amplification index, $\tilde{\Gamma}_{b / 2}^{(k)}(\%)$} \\
\hline & \multicolumn{3}{|c|}{ Fixed base } & \multicolumn{3}{|c|}{ Isolated base } \\
\hline & Storey 1 & Storey 2 & Storey 3 & Storey 1 & Storey 2 & Storey 3 \\
\hline Newhall & $\begin{array}{l}10.50 \\
(31.76)\end{array}$ & $\begin{array}{c}15.43 \\
(31.86)\end{array}$ & $\begin{array}{c}15.56 \\
(31.33)\end{array}$ & $\begin{array}{c}0.79 \\
(22.29)\end{array}$ & $\begin{array}{c}1.51 \\
(24.72)\end{array}$ & $\begin{array}{l}10.57 \\
(26.97)\end{array}$ \\
\hline Synthetic \#1 & $\begin{array}{c}7.50 \\
(39.35)\end{array}$ & $\begin{array}{l}16.90 \\
(41.63)\end{array}$ & $\begin{array}{c}21.33 \\
(41.15)\end{array}$ & $\begin{array}{c}4.36 \\
(27.97)\end{array}$ & $\begin{array}{c}2.95 \\
(29.31)\end{array}$ & $\begin{array}{c}4.51 \\
(29.58)\end{array}$ \\
\hline $2 \times$ El Centro & $\begin{array}{c}0.71 \\
(19.69)\end{array}$ & $\begin{array}{c}0.32 \\
(27.41)\end{array}$ & $\begin{array}{c}2.97 \\
(33.22)\end{array}$ & $\begin{array}{c}1.58 \\
(16.91)\end{array}$ & $\begin{array}{c}0.03 \\
(27.05)\end{array}$ & $\begin{array}{c}1.50 \\
(29.71)\end{array}$ \\
\hline $2 \times$ Melipilla & $\begin{array}{c}18.33 \\
(40.16)\end{array}$ & $\begin{array}{c}21.52 \\
(45.97)\end{array}$ & $\begin{array}{c}23.64 \\
(46.02)\end{array}$ & $\begin{array}{c}0.90 \\
(25.80)\end{array}$ & $\begin{array}{c}0.16 \\
(27.37)\end{array}$ & $\begin{array}{c}12.25 \\
(29.97)\end{array}$ \\
\hline JMA & $\begin{array}{c}9.47 \\
(19.83)\end{array}$ & $\begin{array}{c}10.05 \\
(15.24)\end{array}$ & $\begin{array}{c}9.88 \\
(15.36)\end{array}$ & $\begin{array}{c}0.23 \\
(22.88)\end{array}$ & $\begin{array}{c}0.51 \\
(24.90)\end{array}$ & $\begin{array}{c}4.77 \\
(26.33)\end{array}$ \\
\hline Mean & $\begin{array}{c}9.30 \\
(30.16)\end{array}$ & $\begin{array}{c}12.84 \\
(32.42) \\
12.27(32.00)\end{array}$ & $\begin{array}{c}14.68 \\
(33.41)\end{array}$ & $\begin{array}{c}1.57 \\
(23.17)\end{array}$ & $\begin{array}{c}1.03 \\
(26.67) \\
3.11(26.12)\end{array}$ & $\begin{array}{c}6.72 \\
(28.51)\end{array}$ \\
\hline
\end{tabular}

$(\cdot)$ : Values for the asymmetric structure. 

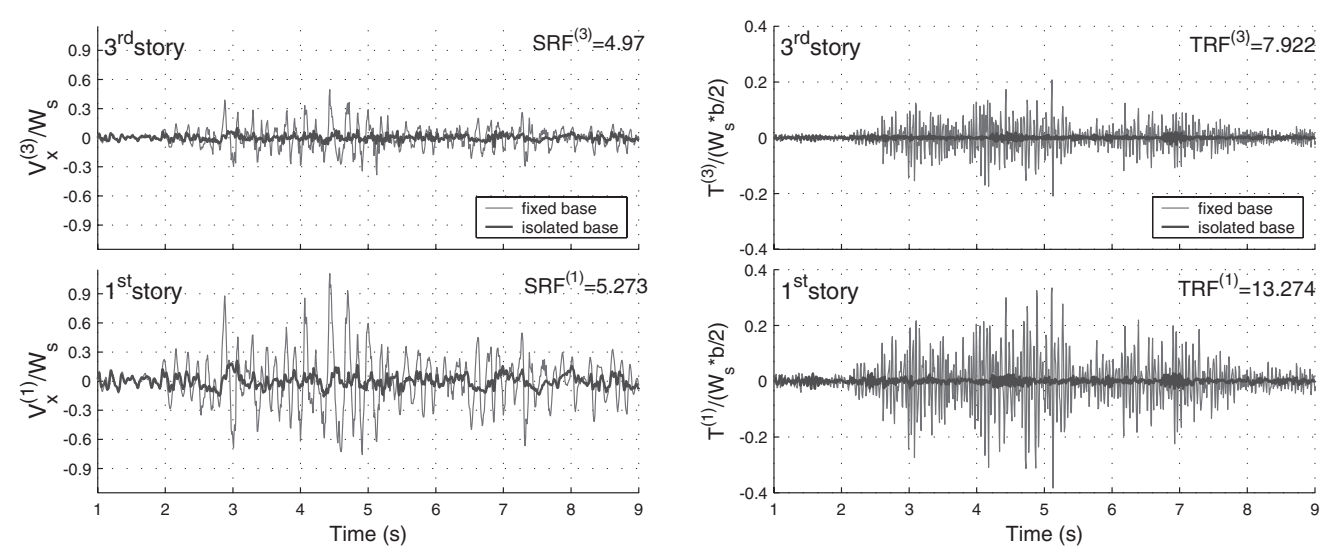

(a) symmetric structure
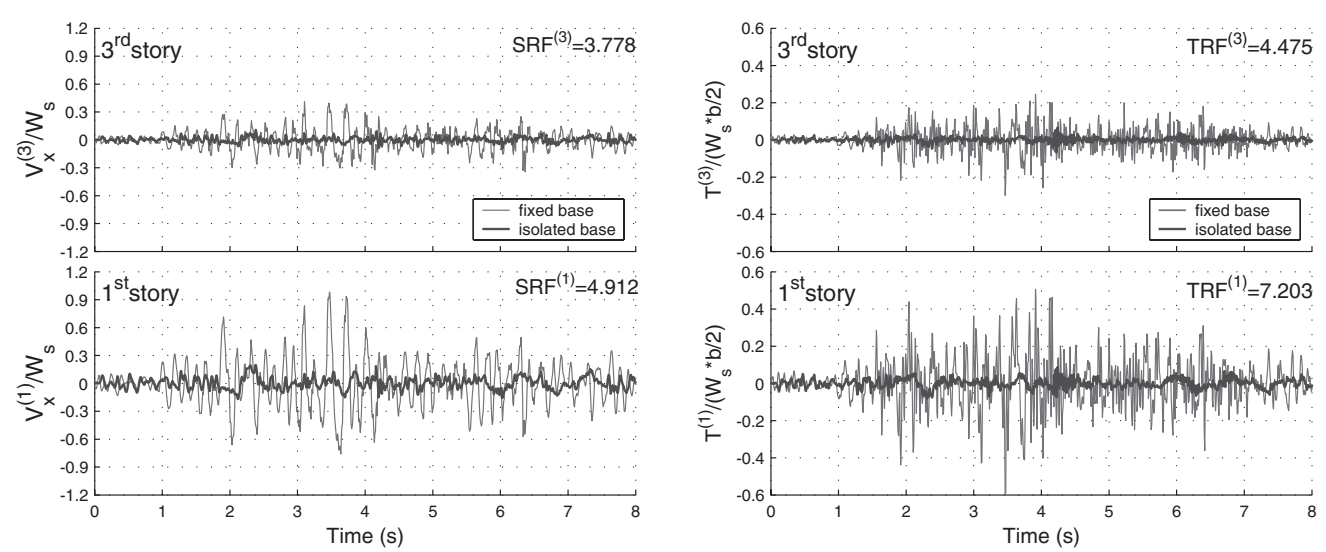

(b) asymmetric structure

Figure 16. Comparison between the experimental shear and torque response histories for the superstructure with fixed-base and base isolation subject to $2 \times$ Melipilla record.

evaluate the reduction in interstorey deformations indirectly through the storey shear $V_{x}^{(j)}$ and torque $T^{(j)}$ by [6]:

$$
\tilde{\Gamma}_{\mp b / 2}^{(j)}=100\left(\frac{\max \left(V_{x}^{(j)} \pm T^{(j)}\left((b / 2) / \Omega_{\mathrm{s}}^{2} \rho_{j}^{2}\right)\right)}{\max \left(V_{x}^{(j)}\right)}-1\right) \quad(\%) \quad j=1: 3
$$

where $\Omega_{\mathrm{s}}$ is the uncoupled torsional to lateral frequency ratio of the structure with fixed base; and $\rho_{j}$ is the radius of gyration of stiffness for the $j$ th storey. This equation, derived for structures with mass asymmetry only, is specially useful for instrumented buildings for which the only measured information is provided by accelerometers.

Finally, shown in Table IV are the torsional amplification factors $\tilde{\Gamma}_{b / 2}^{(j)}=\max \left(\tilde{\Gamma}_{-b / 2}^{(j)}, \tilde{\Gamma}_{+b / 2}^{(j)}\right)$ for the symmetric and asymmetric configuration of the building (Equation (12)). The mean torsional amplification for the three building stories of the fixed-base symmetric building is about $12.3 \%$; the corresponding value for the isolated building is $3.1 \%$, i.e. 4 times smaller. In 
both cases, the maximum amplification occurs in the third storey, due again to the contribution of the higher modes. Analogous results are obtained for the asymmetric model, in which torsional amplifications are more uniform in height as a result of the 'predominant effect' of natural torsion over accidental torsion. Mean amplification values for the fixed-base structure are as large as $32 \%$, decreasing to about $26 \%$ for the isolated configuration, i.e. $23 \%$ smaller.

\section{SUMMARY AND CONCLUSIONS}

The results of this experimental investigation directed to evaluate the torsional behaviour of symmetric and asymmetric structures isolated with the FPS has led to the following conclusions:

(i) For nominally symmetric structures, the measured increase in edge deformations at the base due to accidental torsion is, in the mean, about $2.5 \%$. Such increase in deformations tend to be larger for impulsive ground motions, reaching a peak value of $5.9 \%$. It was shown that the corresponding torsional amplifications of interstorey deformations for the nominally symmetric superstructure may be, in the mean, $1 / 3$ of those corresponding to the fixed-base counterpart. Indeed, torsional amplification factors are usually less than 1.6 for the first two stories, but increase considerably in the third storey as a consequence of higher mode contributions.

(ii) For asymmetric structures, the measured increase in edge deformations at the base due to natural torsion is, in the mean, about $6.2 \%$. Such increase in deformations tend to be larger for impulsive ground motions, reaching a peak value of $10.5 \%$. It was demonstrated that the corresponding torsional amplifications of interstorey deformations for the asymmetric superstructure may be, in the mean, $23 \%$ of those corresponding to the fixed-base counterpart (Table IV). In contrast to the symmetric case, torsional amplification factors are essentially uniform in height.

(iii) These torsional amplification values are considerably smaller, about $45 \%$ for the asymmetric case, than the values predicted by the well known statically derived formula to account for torsional effects present in current codes for isolated structures [19]. In a sequel article, a new formula will be introduced for structures with FPS isolators, since it is a known fact [20] that formulas of torsional amplifications derived from static analysis lead to inconsistent estimates of the true dynamic torsional amplification.

(iv) The experimental identification of the frictional properties of the building model shows that the friction coefficient in the FPS isolators is highly dependent on the deformation velocity and contact pressure in the isolators, a result that has been previously stated [11]. However, experimental results have also shown that the frictional behaviour of the FPS isolation system can be approximately modelled by an associated plastic flow rule. Since the variation in the resultant of the normal loads of all isolators is small, experimental results have shown the 'existence' of a yield surface for the whole FPS system in the symmetric and asymmetric configurations. This implies that a macroelement model as the one presented earlier by the authors $[12,13]$ could be useful in modelling these structures for design purposes.

(v) Measured building responses and their equivalent responses obtained from analytical models of the FPS, such as the physical model presented earlier that includes variability in normal forces, large deformations, sticking, and uplift, show a remarkable similarity 
in their traces and peak values. Thus, the physical model of the FPS can be used effectively to predict the actual earthquake performance of buildings with FPS isolators. Uncertainty in the predictions of peak storey shears is within a one-digit percentage; storey torques in the asymmetric structure are predicted within, say, $20 \%$ accuracy.

(vi) Finally, a comparison between the peak earthquake responses obtained for the fixed and isolated-base configurations of the building has shown that shear reduction factors of 3.9 and 7.3 are expected due to base isolation in the symmetric structure; such factors are 3.9 and 4.8 for the shear and torque, respectively, in the asymmetric case.

\section{ACKNOWLEDGEMENT}

This research has been funded by the Chilean National Research Fund for Science and Technology, Fondecyt, under Grant No. 1000514. The authors are grateful for this support.

\section{REFERENCES}

1. Zayas V, Low S, Mahin S. The FPS earthquake resisting system. Report UCB/EERC-87/01, Earthquake Engineering Research Center, University of California at Berkeley, 1987.

2. Zayas V, Low S, Bozzo L, Mahin S. Feasibility and performance studies on improving the earthquakes resistance of new and existing buildings using the Frictional Pendulum System. Report UCB/EERC-89/09, Earthquake Engineering Research Center, University of California at Berkeley, 1989.

3. Mokha A, Constantinou M, Reinhorn A, Zayas V. Experimental study of Friction Pendulum Isolation System. Journal of Structural Engineering (ASCE) 1991; 116(4):1201-12016.

4. Al-Hussaini T, Zayas V, Constantinou M. Seismic isolation of multi-storey frame structures using spherical sliding isolation system. Report NCEER-94-0007, National Center for Earthquake Engineering Research, State University of New York at Buffalo, 1994.

5. Almazán J, De la Llera J. Physical model for dynamic analysis of structures with FPS isolators. Earthquake Engineering and Structural Dynamics, 2003; 32, in press.

6. Almazán J. Accidental and natural torsion in structures isolated with the Frictional Pendulum System. Doctoral Thesis Dissertation, Pontificia Universidad Católica de Chile, 2001. In Spanish.

7. Stewart D. Platform with six degrees of freedom. Proceedings of the Institution of Mechanical Engineers, vol. 180. Pt. 1. 15, London, England 1965; 371-386.

8. National Instrument Corporation. LabVIEW, User Manual. Bridge Point Parkway, Austin, Texas, 1996.

9. dSPACE digital signal processing and control engineering GmbH. MTRACE Real-time Trace Module for MATLAB, User's Guide. Paderborn, Germany, 1996.

10. Almazán J, De la Llera J. Lateral-torsional coupling of structures isolated with the Frictional Pendulum System. 12th World Conference on Earthquake Engineering, Auckland, New Zealand, 2000.

11. Constantinou M, Mokha A, Reinhorn A. Teflon bearings in base isolation, Part II: modeling. Journal of Structural Engineering (ASCE) 1990; 117:455-474.

12. De la Llera J, Chopra A. A simplified model for analysis and design of asymmetric-plan buildings. Earthquake Engineering and Structural Dynamics 1995; 24(4):573-594.

13. De la Llera J, Vásquez J, Chopra A, Almazán J. A macro-element model for inelastic building analysis. Earthquake Engineering and Structural Dynamics 2000; 29:1725-1757.

14. De la Llera J, Chopra A, Almazán J. Three-dimensional inelastic response of a reinforced concrete building during northridge earthquake. Journal of Structural Engineering (ASCE) 2001; 127(5):1482-489.

15. Almazán J, De la Llera J, Inaudi J. Modeling aspects of structures isolated with the frictional pendulum system. Earthquake Engineering and Structural Dynamics 1998; 27:845-867.

16. Inaudi J, De la Llera J. SAT-Lab: Structural Analysis Toolbox, 2001. www.sat-lab.com, in Spanish.

17. The Mathworks, Inc. MATLAB, Reference Guide. Natick, MA, 1999.

18. De la Llera J, Chopra A. Evaluation of seismic code provisions using strong-motion building records from the 1994 Northridge earthquake. Report UCB/EERC-97/16, Earthquake Engineering Research Center, University of California at Berkeley, 1998.

19. International Code Council, Inc. International Building Code 2000, Falls Church, Virgina, 2000.

20. De la Llera J, Chopra A. Using accidental eccentricity in code-specified static and dynamic analyses of buildings. Earthquake Engineering and Structural Dynamics 1994; 23(9):947-967. 